\title{
Making the Most of Your Samples
}

\author{
Zhiyi Huang* $^{*} \quad$ Yishay Mansour ${ }^{\dagger} \quad$ Tim Roughgarden ${ }^{\ddagger}$
}

\begin{abstract}
We study the problem of setting a price for a potential buyer with a valuation drawn from an unknown distribution $D$. The seller has "data" about $D$ in the form of $m \geq 1$ i.i.d. samples, and the algorithmic challenge is to use these samples to obtain expected revenue as close as possible to what could be achieved with advance knowledge of $D$.

Our first set of results quantifies the number of samples $m$ that are necessary and sufficient to obtain a $(1-\epsilon)$-approximation. For example, for an unknown distribution that satisfies the monotone hazard rate (MHR) condition, we prove that $\tilde{\Theta}\left(\epsilon^{-3 / 2}\right)$ samples are necessary and sufficient. Remarkably, this is fewer samples than is necessary to accurately estimate the expected revenue obtained for such a distribution by even a single reserve price. We also prove essentially tight sample complexity bounds for regular distributions, bounded-support distributions, and a wide class of irregular distributions. Our lower bound approach, which applies to all randomized pricing strategies, borrows tools from differential privacy and information theory, and we believe it could find further applications in auction theory.

Our second set of results considers the single-sample case. While no deterministic pricing strategy is better than $\frac{1}{2}$-approximate for regular distributions, for MHR distributions we show how to do better: there is a simple deterministic pricing strategy that guarantees expected revenue at least 0.589 times the maximum possible. We also prove that no deterministic pricing strategy achieves an approximation guarantee better than $\frac{e}{4} \approx .68$.
\end{abstract}

${ }^{*}$ University of Hong Kong. This research was done while the author was a postdoc at Stanford University. Email: zhiyi@cs.hku.hk.

${ }^{\dagger}$ Microsoft Research and Tel Aviv University. This research was supported in part by The Israeli Centers of Research Excellence (I-CORE) program, (Center No. 4/11), by a grant from the Israel Science Foundation (ISF), by a grant from United States-Israel Binational Science Foundation (BSF), and by a grant from the Israeli Ministry of Science (MoS). Email: mansour@tau.ac.il.

${ }^{\ddagger}$ Stanford University. This research was supported in part by NSF grants CCF-1016885 and CCF-1215965, and an ONR PECASE Award. Email: tim@cs.stanford.edu. 


\section{Introduction}

We study the basic pricing problem of making an "optimal" take-it-or-leave-it price to a potential buyer with an unknown willingness-to-pay (a.k.a. valuation). Offering a price of $p$ to a buyer with valuation $v$ yields revenue $p$ if $v \geq p$, and 0 otherwise. The traditional approach in theoretical computer science to such problems is to assume as little as possible about the buyer's valuation - for example, only lower and upper bounds on its value - and to compare the performance of different prices using worst-case analysis. The traditional approach in economics is to assume that the buyer's valuation is drawn from a distribution $D$ that is known to the seller, and to use average-case analysis. In the latter case, the optimal solution is clear — it is the monopoly price $\max _{p \geq 0} p \cdot(1-F(p))$, where $F$ is the CDF of $D$.

Cole and Roughgarden [9] recently proposed adapting the formalism of learning theory [23] to interpolate between the traditional worst- and average-case approaches, in the context of single-item auction design. The idea is to parameterize a seller's knowledge about an unknown distribution $D$ through a number $m$ of i.i.d. samples from $D$. When $m=0$ this is equivalent to the worst-case approach, and as $m \rightarrow \infty$ it becomes equivalent to the average-case approach. The benchmark is the maximum expected revenue obtainable when the distribution $D$ is known a priori. The algorithmic challenge is to use the $m$ samples from $D$ to get expected revenue as close to this benchmark as possible, no matter what the underlying distribution $D$ is. $^{1}$

This "hybrid" model offers several benefits. First, it is a relatively faithful model of many realistic computer science applications, where data from the past is assumed to be a reasonable proxy for future inputs and guides the choice of an algorithm. For example, in Yahoo!'s keyword auctions, the choice of reserve prices is guided by past bid data in a natural way [19]. Second, the model is a potential "sweet spot" between worst-case and average-case analysis, inheriting much of the robustness of the worst-case model (since we demand guarantees for every underlying $D$ ) while allowing very good approximation guarantees with respect to a strong benchmark. Third, by analyzing the trade-offs between the number of samples $m$ available from $D$ and the best-possible worst-case approximation guarantee, the analysis framework implicitly quantifies the value of data (i.e., of additional samples). It becomes possible, for example, to make statements like "4 times as much data improves our revenue guarantee from $80 \%$ to $90 \%$." Finally, proving positive results in this model involves rigorously justifying natural methods of incorporating past data into an algorithm, and this task is interesting in its own right.

\subsection{Our Results}

Formally, we study a single seller of some good, and a single buyer with a private valuation $v$ for the good drawn from an unknown distribution $D$. The seller has access to $m \geq 1$ i.i.d. samples $v_{1}, \ldots, v_{m}$ from $D$. The goal is to identify, among all $m$-pricing strategies - functions from a sample $v_{1}, \ldots, v_{m}$ to a price $p$ - the strategy that has the highest expected revenue. The expectation here is over $m+1$ i.i.d. draws from $D$ - the samples $v_{1}, \ldots, v_{m}$ and the unknown valuation $v$ of the buyer - and the randomness of the pricing strategy. The approximation guarantee of a pricing strategy $p(\cdot)$ for a set $\mathcal{D}$ of distributions is its worst-case (over $\mathcal{D}$ ) approximation of the (optimal) expected revenue obtained by the monopoly price:

$$
\inf _{D \in \mathcal{D}} \frac{\mathbf{E}_{v_{1}, \ldots, v_{m} \sim D}\left[p\left(v_{1}, \ldots, v_{m}\right) \cdot\left(1-F\left(p\left(v_{1}, \ldots, v_{m}\right)\right)\right)\right]}{\max _{p} p(1-F(p))}
$$

\footnotetext{
${ }^{1}$ There are, of course, other ways one can parameterize partial knowledge about valuations. See e.g. Azar et al. [2], Chiesa et al. [8] for alternative approaches.
} 


\begin{tabular}{|c|ll|ll|}
\hline & \multicolumn{2}{|c|}{ Upper Bound } & \multicolumn{2}{c|}{ Lower Bound } \\
\hline MHR & $O\left(\epsilon^{-3 / 2} \log \epsilon^{-1}\right)$ & $($ Thm. 3.1) & $\Omega\left(\epsilon^{-3 / 2}\right)$ & (Thm. 4.12) \\
Regular & $O\left(\epsilon^{-3} \log \epsilon^{-1}\right)$ & $([11])$ & $\Omega\left(\epsilon^{-3}\right)$ & (Thm. 4.8) \\
General & $O\left(\delta^{-1} \epsilon^{-2} \log \left(\delta^{-1} \epsilon^{-1}\right)\right)$ & (Thm. 3.5) & $\Omega\left(\delta^{-1} \epsilon^{-2}\right)$ & (Thm. 4.6) \\
Bounded Support & $O\left(H \epsilon^{-2} \log \left(H \epsilon^{-1}\right)\right)$ & $($ Thm. 3.6 and [4]) & $\Omega\left(H \epsilon^{-2}\right)$ & (Thm. 4.7) \\
\hline
\end{tabular}

Table 1: Sample complexity of a $(1-\epsilon)$-approximation. For bounded-support distributions, the support is a subset of $[1, H]$. For general distributions, the benchmark is the optimal revenue of prices with sale probability at least $\delta$.

where $F$ is the CDF of $D$.

We first describe our results that quantify the inherent trade-off between the number of samples $m$ and the best-possible approximation guarantee of an $m$-pricing strategy; see also Table 1 . Some restriction on the class $\mathcal{D}$ of allowable distributions is necessary for the existence of pricing strategies with any non-trivial approximation of the optimal expected revenue. ${ }^{2}$ We give essentially tight bounds on the number of samples that are necessary and sufficient to achieve a target approximation of $1-\epsilon$, for all of the choices of the class $\mathcal{D}$ that are common in auction theory. For example, when $\mathcal{D}$ is the set of distributions that satisfy the monotone hazard rate (MHR) condition $^{3}$, we prove that $m=\Omega\left(\epsilon^{-3 / 2}\right)$ samples are necessary and that $m=O\left(\epsilon^{-3 / 2} \log \epsilon^{-1}\right)$ samples are sufficient to achieve an approximation guarantee of $1-\epsilon{ }^{4}$ This bound holds more generally for the class of " $\alpha$-strongly regular distributions" introduced in Cole and Roughgarden [9] (for fixed $\alpha>0$ ). When $\mathcal{D}$ is the (larger) set of regular distributions ${ }^{5}$, we prove that the sample complexity is $\tilde{\Theta}\left(\epsilon^{-3}\right)$. When $\mathcal{D}$ is the set of arbitrary distributions with support contained in $[1, H]$, the sample complexity is $\tilde{\Theta}\left(H \epsilon^{-2}\right)$. We also give essentially optimal sample complexity bounds for distributions that are parameterized by the probability of a sale at the monopoly price; see Section 1.2 for more discussion. On the upper bound side, our primary contribution is the bound for MHR and strongly regular distributions. ${ }^{6}$ All of our lower bounds, which are information-theoretic and apply to arbitrary randomized pricing strategies, are new.

Our second set of results considers the regime where the seller has only one sample $(m=1)$ and wants to use it in the optimal deterministic way. ${ }^{7}$ Dhangwatnotai et al. [11] observed that an elegant result from auction theory, the Bulow-Klemperer theorem on auctions vs. negotiations [6], implies that the 1-sample pricing strategy $p(v)=v$ has an approximation guarantee of $\frac{1}{2}$ when $\mathcal{D}$ is the set of regular distributions. ${ }^{8}$ It is not hard to prove that there is no better deterministic pricing strategy for this set of distributions. We show how to do better, however, when $\mathcal{D}$ is the

\footnotetext{
${ }^{2}$ The family of distributions that take on a value $M$ with probability $\frac{1}{M}$ and 0 with probability $1-\frac{1}{M}$ highlight the difficulty.

${ }^{3} D$ satisfies the monotone hazard condition if $\frac{f(v)}{1-F(v)}$ is nondecreasing; see Section 2 for details.

${ }^{4}$ We suppress only universal constant factors, which do not depend on the specific distribution $D \in \mathcal{D}$. Such uniform sample complexity bounds are desirable because the valuation distribution is unknown. Law of Large Numbers-type arguments do not generally give uniform bounds.

${ }^{5} D$ is regular if the "virtual valuation function" $v-\frac{1-F(v)}{f(v)}$ is non-decreasing; see Section 2 for details.

${ }^{6}$ The upper bound for regular distributions was proved in Dhangwatnotai et al. [11] and the upper bound for bounded valuations can be deduced from Balcan et al. [4].

${ }^{7}$ We offer the problems of determining the best randomized pricing strategy for $m=1$ and the best way to use a small $m \geq 2$ number of samples as challenging and exciting directions for future work.

${ }^{8}$ Dhangwatnotai et al. [11] observed this in the context of the design and analysis of prior-independent auctions. Plugging our better bounds for single-sample pricing starategies with MHR distributions into the framework of Dhangwatnotai et al. [11] immediately yields analogously better prior-independent mechanisms.
} 


\begin{tabular}{|c|l|c|}
\hline & \multicolumn{1}{|c|}{ Positive Result } & \multicolumn{1}{c|}{ Negative Result } \\
\hline Regular & $\geq 0.5 \quad([6,11])$ & $\leq 0.5 \quad$ (Thm. 6.1) \\
MHR & $\geq 0.589($ Thm. 5.1) & $\leq 0.68$ (Thm. 6.3) \\
\hline
\end{tabular}

Table 2: Optimal approximation ratio with a single sample.

smaller set of MHR distributions: a simple 1-pricing strategy of the form $p(v)=c v$ for some $c<1$ has an approximation guarantee of 0.589 . We also prove that no deterministic 1-pricing strategy is better than an $\frac{e}{4}$-approximation for MHR distributions, and that no continuously differentiable such strategy is better than a 0.677-approximation.

\subsection{A Few Technical Highlights}

This section singles out a few of our results and techniques that seem especially useful or motivating for follow-up work. First, recall that we prove that $O\left(\epsilon^{-3 / 2} \log \epsilon^{-1}\right)$ samples from an unknown MHR distribution - or more generally, an unknown $\alpha$-strongly regular distribution [9] — are sufficient to achieve expected revenue at least $1-\epsilon$ times that of the monoply price. Remarkably, this is fewer than the $\approx \epsilon^{-2}$ samples necessary to accurately estimate the expected revenue obtained by even a single fixed price for such a distribution! ${ }^{9}$ In this sense, we prove that near-optimal revenue-maximization is strictly easier than accurately learning even very simple statistics of the underlying distribution. The most important idea in our upper bound is that, because of the structure of the revenue-maximization problem, the estimation errors of different competing prices are usefully correlated. For example, if the estimated expected revenue of the true monopoly price is significantly less than its actual expected revenue (because of a higher-than-expected number of low samples), then this probably also holds for prices that are relatively close to the monopoly price. Moreover, these are precisely the incorrect prices that an algorithm is most likely to choose by mistake. The second ingredient is the fact that MHR distributions have strongly concave "revenue curves," and this limits how many distinct prices can achieve expected revenue close to that of the monopoly price.

Second, recall that we prove essentially matching lower bounds for all of our sample complexity upper bounds. For example, there is no $(1-\epsilon)$-approximate pricing strategy (deterministic or randomized) for MHR distributions when $m=o\left(\epsilon^{-3 / 2}\right)$ or for regular distributions when $m=$ $o\left(\epsilon^{-3}\right)$. For both of these lower bounds, we reduce the existence of a $(1-\epsilon)$-optimal pricing strategy to that of a classifier that distinguishes between two similar distributions. We borrow methodology from the differential privacy literature to construct two distributions with small KL divergence and disjoint sets of near-optimal prices, and use Pinsker's inequality to derive the final sample complexity lower bounds. This lower bound approach is novel in the context of auction theory and we expect it to find further applications.

Third, we offer a simple and novel approach for reasoning about irregular distributions. We noted above the problematic irregular distributions that place a very low probability on a very high value. Regularity can also fail for more "reasonable" distributions, such as mixtures of common distributions. In Section 3.3, we consider a benchmark $R_{\delta}^{*}$ defined as the maximum expected revenue achievable for the underlying distribution using a price that sells with probability at least $\delta$, and

\footnotetext{
${ }^{9}$ It is well known (e.g. Anthony and Bartlett [1, Lemma 5.1]) that, given a coin that either has bias $\frac{1}{2}-\epsilon$ or bias $\frac{1}{2}+\epsilon$, $\Omega\left(\epsilon^{-2} \log \frac{1}{\delta}\right)$ coin flips are necessary to distinguish between the two cases with probability at least $1-\delta$. The lower bound is information-theoretic and applies to arbitrarily sophisticated learning methods. This sample complexity lower bound translates straightforwardly to the problem of estimating, by any means, the expected revenue of a fixed price for an unknown MHR distribution up to a factor of $(1 \pm \epsilon)$.
} 
prove essentially tight sample complexity bounds for approximating this benchmark. As a special case, if for every distribution in $\mathcal{D}$ the monopoly price sells with probability at least $\delta$ - as is the case for sufficiently small $\delta$ and typical "reasonable" distributions, even irregular ones — then approximating $R_{\delta}^{*}$ is equivalent to approximating the optimal revenue. Even if not all distributions of $\mathcal{D}$ satisfy this property, this benchmark enables parameterized sample complexity bounds that do not require blanket distributional restrictions such as regularity. We believe that this parameterized approach will find more applications. ${ }^{10}$

\subsection{Further Related Work}

We already mentioned the related work of Cole and Roughgarden [9]; the present work follows the same formalism. Specializing the results in Cole and Roughgarden [9] to the sample complexity questions that we study here yields much weaker results than the ones we prove - only a lower bound of $\epsilon^{-1 / 2}$ and an upper bound of $\epsilon^{-c}$ for a large constant $c$. Two of the upper bounds in Table 2 follow from previous work. The upper bound of $O\left(\epsilon^{-3} \log \epsilon^{-1}\right)$ for regular distributions was proved in Dhangwatnotai et al. [11]. (They also proved a bound of $O\left(\epsilon^{-2} \log \epsilon^{-1}\right.$ ) for MHR distributions, which is subsumed by our nearly tight bound of $O\left(\epsilon^{-3 / 2} \log \epsilon^{-1}\right)$.) The upper bound of $O\left(H \epsilon^{-2} \log H \epsilon^{-1}\right)$ for bounded valuations can be deduced from Balcan et al. [4]. ${ }^{11}$ We emphasize that, in addition to our new upper bound results in the large-sample regime, there is no previous work on sample complexity lower bounds for our pricing problem nor on the best-possible approximation given a single sample.

There are many less related previous works that also use the idea of independent samples in the context of auction design. For example, some previous works study the asymptotic (in the number of samples) convergence of an auction's revenue to the optimal revenue, without providing any uniform sample complexity bounds. See Neeman [18], Segal [21], Baliga and Vohra [5], and Goldberg et al. [14] for several examples. Some recent and very different uses of samples in auction design include Fu et al. [13], who use samples to extend the Crémer and McLean [10] theorem to partially known valuation distributions, and Chawla et al. [7], who design auctions that both have near-optimal revenue and enable accurate inference about the valuation distribution from samples.

\section{Preliminaries}

Suppose the buyer's value is drawn from a publicly known distribution $D$ whose support is a continuous interval. Let $F$ be the c.d.f. of $D$. If $F$ is differentiable, let $f$ be the p.d.f. of $D$. Let $q(v)=1-F(v)$ be the quantile of value $v$, i.e., the sale probability of reserve price $v$. Let $v(q)$ be the value with quantile $q$.

The first set of distributions we study are those satisfying standard small-tail assumptions such as regularity, monotone hazard rate, and $\alpha$-strong regularity [9]. We explain these assumptions in more detail next. For these distributions, we assume $F$ is differentiable and $f$ exists.

\footnotetext{
${ }^{10}$ See e.g. Hartline and Roughgarden [15, Appendix D], Hartline [17, Chapter 4], and Sivan and Syrgkanis [22] for alternative approaches to parameterizing irregularity.

${ }^{11}$ The paper by Balcan et al. [4] studies a seemingly different problem — the design of digital good auctions with $n$ buyers in a prior-free setting (with bounded valuations). But if one instantiates their model with bidders with i.i.d. valuations from a distribution $D$, then their performance analysis of their RSO mechanism essentially gives a performance guarantee for the empirical monopoly price for $D$ with $n / 2$ samples, relative to the expected revenue of the monopoly price with a single bidder.
} 
Let $R(q)=q v(q)$ be the revenue as a function over the quantile space. We have

$$
R^{\prime}(q)=v(q)+q \frac{d v}{d q}=v-\frac{q(v)}{f(v)}
$$

The virtual valuation function is defined to be $\phi(v)=v-\frac{1-F(v)}{f(v)}=R^{\prime}(q)$. A distribution $D$ is regular if for all value $v$ in its support,

$$
\frac{d \phi}{d v} \geq 0
$$

Note that $v(q)$ is decreasing in $q$. A distribution is regular iff $R^{\prime}(q)=\phi(v)$ is decreasing in $q$ and, thus, $R(q)$ is concave. So $R(q)$ is maximized when $R^{\prime}(q)=\phi(v(q))=0$. Let $q^{*}$ and $v^{*}=v\left(q^{*}\right)$ be the revenue-optimal quantile and reserve price respectively.

A distribution $D$ has monotone hazard rate (MHR) if for all values $v$ in its support,

$$
\frac{d \phi}{d v} \geq 1
$$

Lemma 2.1 (Hartline et al. [16]). For every MHR distribution, $q^{*} \geq \frac{1}{e}$. $(2)$ :

Cole and Roughgarden [9] defined $\alpha$-strong regular distributions to interpolate between (1) and

$$
\frac{d \phi}{d v} \geq \alpha
$$

Many properties of MHR distributions carry over to $\alpha$-strongly regular distributions with different constants. For example:

Lemma 2.2 (Cole and Roughgarden [9]). For any $\alpha$-strongly regular distribution, $q^{*} \geq \alpha^{1 /(1-\alpha)}$.

To reason about general (irregular) distributions, we require an alternative benchmark (recall the Introduction). We propose

$$
R_{\delta}^{*}=\max _{q \geq \delta} q v(q)
$$

the optimal revenue if we only consider reserve prices with sale probability at least $\delta$. Here, we expect the sample complexity to depend on both $\epsilon$ and $\delta$.

\section{$3 \quad$ Asymptotic Upper Bounds}

We now present our positive results in the asymptotic regime.

Definition 3.1. Given $m$ samples $v_{1} \geq v_{2} \geq \cdots \geq v_{m}$, the empirical reserve is

$$
\underset{i \geq 1}{\arg \max } i \cdot v_{i}
$$

If we only consider $i \geq \mathrm{cm}$ for some parameter $c$, it is called the $c$-guarded empirical reserve. 


\subsection{MHR Upper Bound}

We next prove the following.

Theorem 3.1. The empirical reserve with $m=\Theta\left(\epsilon^{-3 / 2} \log \epsilon^{-1}\right)$ samples is $(1-\epsilon)$-approximate for all MHR distributions.

We also give a matching lower bound (up to the log factor) in Section 4.

For simplicity of presentation, we prove Theorem 3.1 for the $\frac{1}{e}$-guarded empirical reserve. (Recall that $q^{*} \geq \frac{1}{e}$ for MHR distributions.) The unguarded version is similar but requires some extra care on the small quantiles.

To show Theorem 3.1, we use two properties of MHR distributions. First, the optimal quantile of an MHR distribution is at least $e^{-1}$ (Lemma 2.1). Second, the revenue decreases quadratically in how much the reserve price deviates from the optimal one in quantile space, which we formulate as the following lemma.

Lemma 3.2. For any $0 \leq q^{\prime} \leq 1$, we have $R\left(q^{*}\right)-R\left(q^{\prime}\right) \geq \frac{1}{4}\left(q^{*}-q^{\prime}\right)^{2} R\left(q^{*}\right)$.

Proof. There are three cases depending on the relation between $q^{\prime}$ and $q^{*}: q^{\prime}>q^{*}, q^{\prime}=q^{*}$, and $q^{\prime}<q^{*}$. The second case, i.e., $q^{\prime}=q^{*}$, is trivial. Next, we prove the other two cases separately.

First, consider the case when $q^{\prime}>q^{*}$. By the optimality of $q^{*}$, for any $q$ s.t. $q^{*} \leq q \leq q^{\prime}$, we have $q v(q) \leq q^{*} v\left(q^{*}\right)$ and, thus,

$$
v(q) \leq \frac{q^{*}}{q} v\left(q^{*}\right)
$$

Further, by the MHR assumption that $\frac{d \phi(v)}{d v} \geq 1$, for any $q^{*} \leq q \leq q^{\prime}$, we have

$$
\phi(v(q)) \leq \phi\left(v\left(q^{*}\right)\right)+v(q)-v\left(q^{*}\right)=v(q)-v\left(q^{*}\right)
$$

Combining with the above inequality that lower bounds $v(q)$, we get that

$$
\phi(v(q)) \leq \frac{q^{*}-q}{q} v\left(q^{*}\right)
$$

Therefore, we get that

$$
R\left(q^{*}\right)-R\left(q^{\prime}\right)=\int_{q^{*}}^{q^{\prime}}-R^{\prime}(q) d q=\int_{q^{*}}^{q^{\prime}}-\phi(v(q)) d q \geq \int_{q^{*}}^{q^{\prime}} \frac{q-q^{*}}{q} v\left(q^{*}\right) d q
$$

Note that $\frac{q-q^{*}}{q} \geq 0$ for any $q^{\prime} \leq q \leq q^{*}$. Moreover, for any $q \geq \frac{q^{\prime}+q^{*}}{2}$, we have $\frac{q-q^{*}}{q} \geq \frac{q^{\prime}-q^{*}}{q^{\prime}+q^{*}}$. Hence, we further drive the following inequality

$$
R\left(q^{*}\right)-R\left(q^{\prime}\right) \geq \int_{\frac{q^{\prime}+q^{*}}{2}}^{q^{\prime}} \frac{q^{\prime}-q^{*}}{q^{\prime}+q^{*}} v\left(q^{*}\right) d q=\frac{\left(q^{\prime}-q^{*}\right)^{2}}{2\left(q^{\prime}+q^{*}\right)} v\left(q^{*}\right)=\frac{\left(q^{\prime}-q^{*}\right)^{2}}{2 q^{*}\left(q^{\prime}+q^{*}\right)} R\left(q^{*}\right) .
$$

Then lemma then follows from that $0 \leq q^{\prime}, q^{*} \leq 1$.

Next, we consider the case when $q^{\prime}<q^{*}$. The high-level proof idea of this case is similar to the previous case, but requires some subtle changes in the inequalities. For completeness, we include the proof below

By concavity of the revenue curve, for any $q^{\prime} \leq q \leq q^{*}$, we have

$$
q v(q) \geq \frac{q-q^{\prime}}{q^{*}-q^{\prime}} q^{*} v\left(q^{*}\right)+\frac{q^{*}-q}{q^{*}-q^{\prime}} q^{\prime} v\left(q^{\prime}\right) .
$$


Dividing both sides by $q$, we have

$$
v(q) \geq \frac{q^{*} v\left(q^{*}\right)-q^{\prime} v\left(q^{\prime}\right)}{q^{*}-q^{\prime}}+\frac{q^{*} q^{\prime}}{q\left(q^{*}-q^{\prime}\right)}\left(v\left(q^{\prime}\right)-v\left(q^{*}\right)\right) .
$$

Further, by the MHR assumption,

$$
\phi(v(q)) \geq \phi\left(v\left(q^{*}\right)\right)+v(q)-v\left(q^{*}\right)=v(q)-v\left(q^{*}\right) .
$$

Note that the direction of the inequality is the opposite of its counterpart in the previous case. This is because we have $v(q)>v\left(q^{*}\right)$ in this case (as oppose to $v(q)<v\left(q^{*}\right)$ as in the previous case.) Combining with the above inequality that lower bounds $v(q)$, we get that

$$
\begin{aligned}
\phi(v(q)) & \geq \frac{q^{*} v\left(q^{*}\right)-q^{\prime} v\left(q^{\prime}\right)}{q^{*}-q^{\prime}}+\frac{q^{*} q^{\prime}}{q\left(q^{*}-q^{\prime}\right)}\left(v\left(q^{\prime}\right)-v\left(q^{*}\right)\right)-v\left(q^{*}\right) \\
& =\frac{q^{\prime}\left(q^{*}-q\right)}{q\left(q^{*}-q^{\prime}\right)}\left(v\left(q^{\prime}\right)-v\left(q^{*}\right)\right) \geq \frac{q^{\prime}\left(q^{*}-q\right)}{q^{*}\left(q^{*}-q^{\prime}\right)}\left(v\left(q^{\prime}\right)-v\left(q^{*}\right)\right),
\end{aligned}
$$

where the last inequality is due to $q \leq q^{*}$. Hence, we have

$$
\begin{aligned}
R\left(q^{*}\right)-R\left(q^{\prime}\right) & =\int_{q^{\prime}}^{q^{*}} R^{\prime}(q) d q=\int_{q^{\prime}}^{q^{*}} \phi(v(q)) d q \geq \int_{q^{\prime}}^{q^{*}} \frac{q^{\prime}\left(q^{*}-q\right)}{q^{*}\left(q^{*}-q^{\prime}\right)}\left(v\left(q^{\prime}\right)-v\left(q^{*}\right)\right) d q \\
& =\frac{q^{\prime}}{2 q^{*}}\left(q^{*}-q^{\prime}\right)\left(v\left(q^{\prime}\right)-v\left(q^{*}\right)\right) .
\end{aligned}
$$

On the other hand, we have

$$
R\left(q^{*}\right)-R\left(q^{\prime}\right)=q^{*} v\left(q^{*}\right)-q^{\prime} v\left(q^{\prime}\right) .
$$

Taking the linear combination $\frac{2 q^{*}}{3 q^{*}-q^{\prime}} \cdot(4)+\frac{q^{*}-q^{\prime}}{3 q^{*}-q^{\prime}} \cdot(5)$, we have

$$
R\left(q^{*}\right)-R\left(q^{\prime}\right) \geq \frac{\left(q^{*}-q^{\prime}\right)^{2}}{3 q^{*}-q^{\prime}} v\left(q^{*}\right)=\frac{1}{q^{*}\left(3 q^{*}-q^{\prime}\right)}\left(q^{*}-q^{\prime}\right)^{2} R\left(q^{*}\right) \geq \frac{1}{3}\left(q^{*}-q^{\prime}\right)^{2} R\left(q^{*}\right),
$$

where the last inequality holds because $0 \leq q^{*}, q^{\prime} \leq 1$.

Next we show how to use the lemma (and additional ideas) to prove Theorem 3.1.

Proof of Theorem 3.1. We first show that for any two samples $v_{1}$ and $v_{2}$ with quantiles $q_{1}, q_{2}$ such that either $q_{1}<q_{2}<q^{*}$ or $q^{*}<q_{1}<q_{2}$, if the revenue of one of them is at least $\left(1-\frac{\epsilon}{2}\right)$ times smaller than that of the other, e.g., $q_{1}<q_{2}<q^{*}$ and $v_{1} q_{1}<(1-\epsilon) v_{2} q_{2}$, then with probability at least $1-o\left(\frac{1}{m^{2}}\right)$ the algorithm would choose $v_{2}$ over $v_{1}$. Further, with high probability, there is at least one sample that is $\frac{\epsilon}{2}$-close to $q^{*}$ in quantile space both among samples with quantile at least $q^{*}$ and among those with quantile at most $q^{*}$. By concavity of the revenue curve, such samples are $\left(1-\frac{\epsilon}{2}\right)$-optimal. So the theorem follows from union bound.

Let us focus on the case when $q_{1}<q_{2}<q^{*}$ and $v_{1} q_{1}<(1-\epsilon) v_{2} q_{2}$ as the other case is almost identical. Suppose $R\left(q_{1}\right)=(1-\Delta) R\left(q_{2}\right)$ and $q_{1}=q_{2}-\delta$. By concavity of the revenue curve and Lemma 3.2, we have

$$
R\left(q_{2}\right)-R\left(q_{1}\right) \geq R\left(q^{*}\right)-R\left(q^{*}-q_{2}+q_{1}\right) \geq \frac{1}{4}\left(q_{2}-q_{1}\right)^{2} R\left(q^{*}\right) \geq \frac{1}{4}\left(q_{2}-q_{1}\right)^{2} R\left(q_{2}\right) .
$$


So we have $\Delta=\Omega\left(\delta^{2}\right)$.

Let $\tilde{q}_{i} m$ be the number of samples with value at least $v_{i}, i=1,2$. The goal is to show that $\tilde{q}_{1} v_{1}<\tilde{q}_{2} v_{2}$ with probability at least $1-o\left(\frac{1}{m^{2}}\right)$.

Note that the straightforward argument does not work because that we would need $\tilde{\Omega}\left(\epsilon^{-2}\right)$ samples to estimate $q_{i}$ up to a $(1-\epsilon)$ factor. Before diving into the technical proof, let us explain informally how to get away with fewer samples. A bad scenario for the straightforward argument is when, say, $\tilde{q}_{1}>(1+\Delta) q_{1}$ and $\tilde{q}_{2}<(1-\Delta) q_{2}$. We observe that such a bad scenario is very unlikely due to correlation between $\tilde{q}_{1}$ and $\tilde{q}_{2}$ : the samples used to estimate $q_{1}$ and $q_{2}$ are the same; those that cause the algorithm to overestimate $q_{1}$ also contribute to the estimation of $q_{2}$; for the bad scenario to happen, it must be that the number of samples $q_{1}$ and $q_{2}$ is much smaller than its expectation (as we will formulate as (6)), whose probability is tiny.

Now we proceed with the formal proof. Since we consider the $\frac{1}{e}$-guarded empirical reserve, $q_{1}, q_{2} \geq \frac{1}{e}$. By the Chernoff bound, with $\Theta\left(\epsilon^{-3 / 2} \log \epsilon^{-1}\right)$ samples, we have $\tilde{q}_{i} \geq\left(1-\epsilon^{3 / 4}\right) q_{i}=\Omega(1)$ and $\tilde{q}_{i} \leq\left(1+\epsilon^{3 / 4}\right) q_{i}$ with high probability.

If $\tilde{q}_{1} v_{1} \geq \tilde{q}_{2} v_{2}$, then

$$
\frac{\tilde{q}_{1}}{\tilde{q}_{2}} \geq \frac{v_{2}}{v_{1}}=\frac{R\left(q_{2}\right)}{R\left(q_{1}\right)} \frac{q_{1}}{q_{2}}=(1-\Delta)^{-1} \frac{q_{1}}{q_{2}}=\frac{q_{1}}{q_{2}}+\Omega(\Delta) .
$$

So

$$
\begin{aligned}
\tilde{q}_{2}-\tilde{q}_{1} & =\left(1-\frac{\tilde{q}_{1}}{\tilde{q}_{2}}\right) \tilde{q}_{2} \leq\left(1-\frac{\tilde{q}_{1}}{\tilde{q}_{2}}\right)\left(1+\epsilon^{3 / 4}\right) q_{2} \\
& \leq\left(1-\frac{q_{1}}{q_{2}}-\Omega(\Delta)\right)\left(1+\epsilon^{3 / 4}\right) q_{2}=q_{2}-q_{1}+\delta \epsilon^{3 / 4}-\Omega(\Delta) .
\end{aligned}
$$

Since $\delta=O(\sqrt{\Delta})$ and $\Delta \geq \frac{\epsilon}{2}$, we have $\delta \epsilon^{3 / 4}=o(\Delta)$. So

$$
\tilde{q}_{2}-\tilde{q}_{1} \leq q_{2}-q_{1}-\Omega(\Delta)
$$

That is, the number of samples that fall between $q_{1}$ and $q_{2}$ is smaller than its expectation by at least $\Omega(\Delta m)$. By the Chernoff bound, the probability of this event is at most $\exp \left(-\frac{\Delta^{2} m}{\delta}\right)$. Recall that $\Delta=\Omega\left(\delta^{2}\right), \Delta \geq \frac{\epsilon}{2}$, and $m=\Theta\left(\epsilon^{-3 / 2} \log \epsilon^{-1}\right)$. So this probability is at most $\exp \left(-\Omega\left(\log \epsilon^{-1}\right)\right)=$ $o\left(\frac{1}{m^{2}}\right)$ with an appropriate choice of parameters.

\section{$3.2 \alpha$-Strongly Regular Upper Bound}

Our proof of Theorem 3.1 can be extended to $\alpha$-strongly regular distributions with $\alpha>0$. We present the formal statement and sketch the necessary changes below.

Theorem 3.3. The empirical reserve with $m=\Theta\left(\epsilon^{-3 / 2} \log \epsilon^{-1}\right)$ samples is $(1-\epsilon)$-approximate for all $\alpha$-strongly regular distributions, for a constant $\alpha>0$.

The proof of Theorem 3.1 relies on two properties: the monopoly price having at least constant sale probability (Lemma 2.1), and strict concavity of the revenue curve at the monopoly quantile (Lemma 3.2). The proof of Theorem 3.3 is identical, modulo using weaker versions of the lemmas. Specifically, we will replace Lemma 2.1 by Lemma 2.2, and Lemma 3.2 by the following lemma, whose proof is almost identical to that of Lemma 3.2.

Lemma 3.4. For any $q^{\prime} \neq q^{*}, R\left(q^{*}\right)-R\left(q^{\prime}\right) \geq \frac{\alpha}{3}\left(q^{*}-q^{\prime}\right)^{2} R\left(q^{*}\right)$.

In the Appendix we use Lemma 3.4 to prove Theorem 3.3. 


\subsection{General Upper Bounds}

Next, we present a sample complexity upper bound for general distributions using $R_{\delta}^{*}$ as a benchmark. Recall that $R_{\delta}^{*}$ is the optimal revenue by prices with sale probability at least $\delta$.

Theorem 3.5. The $\frac{\delta}{2}$-guarded empirical reserve with $m=\Theta\left(\delta^{-1} \epsilon^{-2} \log \left(\delta^{-1} \epsilon^{-1}\right)\right)$ gives revenue at least $(1-\epsilon) R_{\delta}^{*}$ for all distributions.

Proof Sketch. The proof is standard so we present only a sketch here. Let $q_{\delta}^{*}=\arg \max _{q \geq \delta} q v(q)$ be the optimal reserve price with sale probability at least $\delta$. With high probability, there exists at least one sampled price with quantile between $\left(1-\frac{\epsilon}{3}\right) q^{*}$ and $q^{*}$ : this price has revenue at least $\left(1-\frac{\epsilon}{3}\right) R_{\delta}^{*}$. Further, since $q^{*} \geq \delta$, this price has rank at least $\frac{\delta}{2}$ (among sampled prices) with high probability and thus is considered by the empirical reserve algorithm. Finally, with high probability, any sampled price with rank at least $\frac{\delta}{2}$ has sale probability at least $\frac{\delta}{4}$; for prices with sale probability at least $\frac{\delta}{4}$, the algorithm estimates their sale probability up to a $1-\frac{\epsilon}{3}$ factor with high probability with $m=\Theta\left(\delta^{-1} \epsilon^{-2} \log \left(\delta^{-1} \epsilon^{-1}\right)\right)$ samples. The theorem then follows.

We remark that one can also derive a bound for a single sample (i.e., $m=1$ ) which guarantees expected revenue at least $(\delta / 2) R_{\delta}^{*}$.

Note that for distributions with support $[1, H]$, the optimal sale probability is at least $1 / H$. So we have the following theorem as a direct corollary of Theorem 3.5. This bound can also be deduced from [4]; we include it for completeness.

Theorem 3.6. The empirical reserve with $m=\Theta\left(H \epsilon^{-2} \log \left(H \epsilon^{-1}\right)\right)$ samples is $(1-\epsilon)$-approximate for all distributions with support $[1, H]$.

\section{Asymptotic Lower Bounds}

This section gives asymptotically tight (up to a log factor) sample complexity lower bounds. These lower bounds are information-theoretic and apply to all possible pricing strategies, including randomized strategies. We first present a general framework for proving sample complexity lower bounds, and then instatiate it for each of the classes of distributions listed in Table 1.

\subsection{Lower Bound Framework: Reducing Pricing to Classification}

The high-level plan is to reduce the pricing problem to a classification problem. We will construct two distributions $D_{1}$ and $D_{2}$ and show that given any pricing algorithm that is $(1-\epsilon)$-approximate for both $D_{1}$ and $D_{2}$, we can construct a classification algorithm that can distinguish $D_{1}$ and $D_{2}$ with constant probability, say, $\frac{1}{3}$, using the same number of samples as the pricing algorithm. Further, we will construct $D_{1}$ and $D_{2}$ to be similar enough and use tools from information theory to show a lower bound on the number of samples needed to distinguish the two distributions.

Information Theory Preliminaries Consider two distributions $P_{1}$ and $P_{2}$ over a sample space $\Omega$. Let $p_{1}$ and $p_{2}$ be the density functions. The statistical distance between $P_{1}$ and $P_{2}$ is:

$$
\delta\left(P_{1}, P_{2}\right)=\frac{1}{2} \int_{\Omega}\left|p_{1}(\omega)-p_{2}(\omega)\right| d \omega
$$

In information theory, it is known (e.g., Baigneres et al. [3]) that no classification algorithm $A: \Omega \rightarrow\{1,2\}$ can distinguish $P_{1}$ and $P_{2}$ correctly with probability strictly better than $\frac{\delta\left(P_{1}, P_{2}\right)+1}{2}$, 
i.e., there exists $i \in\{1,2\}, \operatorname{Pr}_{\omega \sim P_{i}}[A(\omega)=i] \leq \frac{\delta\left(P_{1}, P_{2}\right)+1}{2}$. This lower bound applies to arbitrary randomized classification algorithms.

Suppose we want to show a sample complexity lower bound of $m$. Then we will let $P_{i}=D_{i}^{m}$ and upper bound $\delta\left(P_{1}, P_{2}\right)$. However, the statistical distance is hard to bound directly when we have multiple samples: $\delta\left(D_{1}^{m}, D_{2}^{m}\right)$ cannot be written as function of $m$ and $\delta\left(D_{1}, D_{2}\right)$. In particular, the statistical distance does not grow linearly with the number of samples.

In order to derive an upper bound on the statistical distance with multiple samples, it is many times convenient to use the Kullback-Leibler $(K L)$ divergence, which is defined as follows:

$$
D_{K L}\left(P_{1} \| P_{2}\right)=\mathbf{E}_{\omega \sim P_{1}}\left[\ln \frac{p_{1}(\omega)}{p_{2}(\omega)}\right] .
$$

In information theory, the KL divergence can be viewed as the redundancy in the encoding in the case that the true distribution is $P_{1}$ and we use the optimal encoding for distribution $P_{2}$. One nice property of the KL divergence is that it is additive over samples: if $P_{1}=D_{1}^{m}$ and $P_{2}=D_{2}^{m}$ are the distributions over $m$ samples of $D_{1}$ and $D_{2}$, then the $K L$ divergence of $P_{1}$ and $P_{2}$ is $m$ times $D_{K L}\left(D_{1} \| D_{2}\right)$.

We can relate the KL divergence to the statistical distance through Pinsker's inequality [20], which states that:

$$
\delta\left(P_{1}, P_{2}\right) \leq \sqrt{\frac{1}{2} D_{K L}\left(P_{1} \| P_{2}\right)}
$$

By symmetry, we also have $\delta\left(P_{1}, P_{2}\right) \leq \sqrt{\frac{1}{2} D_{K L}\left(P_{2} \| P_{1}\right)}$, so

$$
\delta\left(P_{1}, P_{2}\right) \leq \frac{1}{2} \sqrt{D_{K L}\left(P_{1} \| P_{2}\right)+D_{K L}\left(P_{2} \| P_{1}\right)} .
$$

This implies that we can upper bound the statistical distance of $m$ samples from $D_{1}$ and $D_{2}$ by $\frac{1}{2} \sqrt{m \cdot\left(D_{K L}\left(D_{1} \| D_{2}\right)+D_{K L}\left(D_{2} \| D_{1}\right)\right)}$. To get statistical distance at least, say, $\frac{1}{3}$, we need $m=\frac{4}{9} \frac{1}{D_{K L}\left(D_{1} \| D_{2}\right)+D_{K L}\left(D_{2} \| D_{1}\right)}$ samples.

Reducing Pricing to Classification Next, we present the reduction from pricing to classification. Given a value distribution $D$ and $\alpha<1$, its $\alpha$-optimal price set is defined to be the set of reserve prices that induce at least $\alpha$ fraction of the optimal revenue.

Lemma 4.1. If value distributions $D_{1}$ and $D_{2}$ have disjoint $(1-3 \epsilon)$-approximate price sets, and there is a pricing algorithm that is $(1-\epsilon)$-approximate for both $D_{1}$ and $D_{2}$, then there is an classification algorithm that distinguish $P_{1}$ and $P_{2}$ correctly with probability at least $\frac{2}{3}$, using the same number of samples as the pricing algorithm.

We omit the straightforward proof. Note that to distinguish $P_{1}$ and $P_{2}$ correctly with probability at least $\frac{2}{3}$, the statistical distance between $P_{1}$ and $P_{2}$ is least $\frac{1}{3}$. So we have the following theorem.

Theorem 4.2. If value distributions $D_{1}$ and $D_{2}$ have disjoint $(1-3 \epsilon)$-approximate price sets, and there is a pricing algorithm that is $(1-\epsilon)$-approximate for both $D_{1}$ and $D_{2}$, then the algorithm uses at least $\frac{4}{9} \frac{1}{D_{K L}\left(D_{1} \| D_{2}\right)+D_{K L}\left(D_{2} \| D_{1}\right)}$ samples.

A Tool for Constructing Distributions with Small KL Divergence Given Theorem 4.2, our goal is to construct a pair of distributions with small relative entropy subject to having disjoint approximately optimal price sets. Here we introduce a lemma from the differential privacy literature that is useful for constructing pairs of distributions with small KL divergence. 
Lemma 4.3 (Lemma III.2 of Dwork et al. [12]). If distributions $D_{1}$ and $D_{2}$ with densities $f_{1}$ and $f_{2}$ satisfy that $(1+\epsilon)^{-1} \leq \frac{f_{1}(\omega)}{f_{2}(\omega)} \leq(1+\epsilon)$ for every $\omega \in \Omega$, then

$$
D_{K L}\left(D_{1} \| D_{2}\right)+D_{K L}\left(D_{2} \| D_{1}\right) \leq \epsilon^{2} .
$$

For completeness, we include the proof in the Appendix.

The following two useful variants have similar proofs.

Lemma 4.4. If distributions $D_{1}$ and $D_{2}$ satisfy the condition in Lemma 4.3, and further there is a subset of outcomes $\Omega^{\prime}$ such that $p_{1}(\omega)=p_{2}(\omega)$ for every $\omega \in \Omega^{\prime}$, then

$$
D_{K L}\left(D_{1} \| D_{2}\right)+D_{K L}\left(D_{2} \| D_{1}\right) \leq \epsilon^{2}\left(1-p_{1}\left(\Omega^{\prime}\right)\right)
$$

Lemma 4.5. If distributions $D_{1}$ and $D_{2}$ satisfy that $(1+\epsilon)^{-1} \leq \frac{f_{1}(\omega)}{f_{2}(\omega)} \leq(1+\epsilon)$ for every $\omega \in \Omega$ and $\left(1+\epsilon^{\prime}\right)^{-1} \leq \frac{f_{1}(\omega)}{f_{2}(\omega)} \leq\left(1+\epsilon^{\prime}\right)$ for any $\omega \in \Omega^{\prime} \subseteq \Omega$, then

$$
D_{K L}\left(D_{1} \| D_{2}\right)+D_{K L}\left(D_{2} \| D_{1}\right) \leq \epsilon^{2} p_{1}\left(\Omega \backslash \Omega^{\prime}\right)+\left(\epsilon^{\prime}\right)^{2} p_{1}\left(\Omega^{\prime}\right) .
$$

\subsection{Applications}

Inspired by the above lemmas, we will aim to construct $D_{1}$ and $D_{2}$ such that the densities of all values are close in the two distributions.

General Lower Bound As a warm-up case, we demonstrate how to use the above framework to derive a tight (up to a log factor) sample complexity lower bound for general distributions using $R_{\delta}^{*}$ as benchmark. Recall that $R_{\delta}^{*}$ is the optimal revenue by prices with sale probability at least $\delta$.

Theorem 4.6. Every pricing algorithm that guarantees at least $(1-\epsilon) R_{\delta}^{*}$ revenue for all distributions uses at least $\Omega\left(\delta^{-1} \epsilon^{-2}\right)$ samples.

Proof. Let $D_{1}$ and $D_{2}$ be two distributions with support $\left\{H=\delta^{-1}, 2,1\right\}: D_{1}$ takes value $H$ with probability $\frac{1+3 \epsilon}{H}, 2$ with probability $\frac{1-3 \epsilon}{H}$, and 1 with probability $1-\frac{2}{H} ; D_{2}$ takes value $H$ with probability $\frac{1-3 \epsilon}{H}, 2$ with probability $\frac{1+3 \epsilon}{H}$, and 1 with probability $1-\frac{2}{H}$. Clearly, $D_{1}$ and $D_{2}$ have disjoint $(1-3 \epsilon)$-approximate price sets. Further,

$$
D_{K L}\left(D_{1} \| D_{2}\right)=D_{K L}\left(D_{2} \| D_{1}\right)=\frac{1+3 \epsilon}{H} \ln \frac{1+3 \epsilon}{1-3 \epsilon}+\frac{1-3 \epsilon}{H} \ln \frac{1-3 \epsilon}{1+3 \epsilon}=\frac{6 \epsilon}{H} \ln \frac{1+3 \epsilon}{1-3 \epsilon}=O\left(\frac{\epsilon^{2}}{H}\right) .
$$

So the claim follows from Theorem 4.2.

The way we prove Theorem 4.6 also implies a tight (up to a log factor) sample complexity lower bound for distributions with support in $[1, H]$.

Theorem 4.7. Any pricing algorithm that is $(1-\epsilon)$-approximate for all distributions with support $[1, H]$ uses at least $\Omega\left(H \epsilon^{-2}\right)$ samples. 


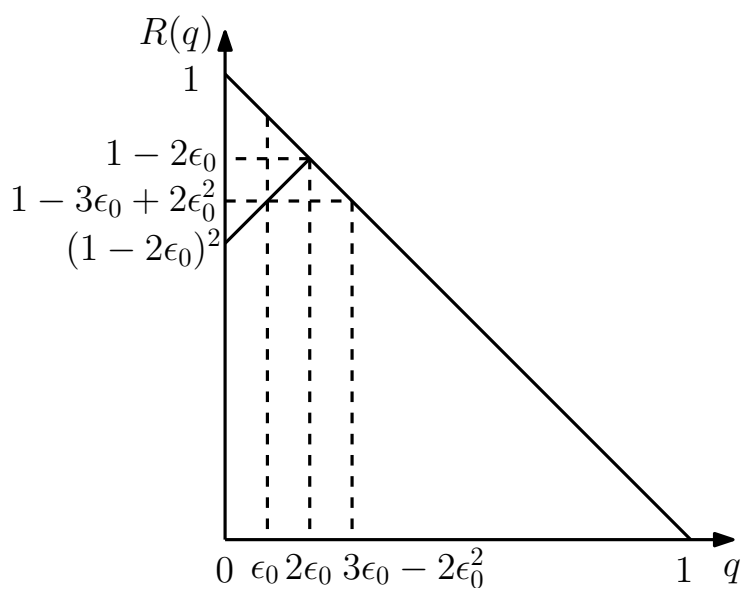

Figure 1: $D_{1}$ is the distribution with revenue curve $R_{1}$ that goes from $(0,1)$ to $(1,0) . D_{2}$ is identical to $D_{1}$ for quantiles from $2 \epsilon_{0}$ to 1 ; for quantiles from 0 to $2 \epsilon_{0}, D_{2}$ 's revenue curve goes from $\left(0,\left(1-2 \epsilon_{0}\right)^{2}\right)$ to $\left(2 \epsilon_{0}, 1-2 \epsilon_{0}\right)$.

Regular Lower Bound We now show that $(1-\epsilon)$-approximate pricing for regular distributions requires $\Omega\left(\epsilon^{-3}\right)$ samples.

Theorem 4.8. Any pricing algorithm that is $(1-\epsilon)$-approximate for all regular distributions uses at least $\frac{(1-6 \epsilon)^{2}}{486 \epsilon^{3}}=\Omega\left(\frac{1}{\epsilon^{3}}\right)$ samples.

This result implies, for example, we need at least 12 samples to guarantee 95 percent of the optimal revenue, and at least 1935 samples to guarantee 99 percent.

We next describe the two distributions that we use and explain the lower bound for regular distributions.Let $D_{1}$ be the distribution with c.d.f. $F_{1}(v)=1-\frac{1}{v+1}$ and p.d.f. $f_{1}(v)=\frac{1}{(v+1)^{2}}$. Let $\epsilon_{0}=3 \epsilon$ and let $D_{2}$ be the distribution with c.d.f.

$$
F_{2}(v)= \begin{cases}1-\frac{1}{v+1} & \text { if } 0 \leq v \leq \frac{1-2 \epsilon_{0}}{2 \epsilon_{0}} \\ 1-\frac{\left(1-2 \epsilon_{0}\right)^{2}}{v-\left(1-2 \epsilon_{0}\right)} & \text { if } v>\frac{1-2 \epsilon_{0}}{2 \epsilon_{0}}\end{cases}
$$

and p.d.f.

$$
f_{2}(v)= \begin{cases}\frac{1}{(v+1)^{2}} & \text { if } 0 \leq v \leq \frac{1-2 \epsilon_{0}}{2 \epsilon_{0}} \\ \frac{\left(1-2 \epsilon_{0}\right)^{2}}{\left(v-\left(1-2 \epsilon_{0}\right)\right)^{2}} & \text { if } v>\frac{1-2 \epsilon_{0}}{2 \epsilon_{0}}\end{cases}
$$

We have

$$
\frac{f_{1}}{f_{2}}= \begin{cases}1 & \text { if } 0 \leq v \leq \frac{1-2 \epsilon_{0}}{2 \epsilon_{0}} \\ \frac{1}{\left(1-2 \epsilon_{0}\right)^{2}} \frac{\left(v-\left(1-2 \epsilon_{0}\right)\right)^{2}}{(v+1)^{2}} \in\left[\left(1-2 \epsilon_{0}\right)^{2},\left(1-2 \epsilon_{0}\right)^{-2}\right] & \text { if } v>\frac{1-2 \epsilon_{0}}{2 \epsilon_{0}} .\end{cases}
$$

The revenue curves of $D_{1}$ and $D_{2}$ are summarized in Figure 1 .

Lemma 4.9.

$$
D_{K L}\left(D_{1} \| D_{2}\right)+D_{K L}\left(D_{2} \| D_{1}\right) \leq \frac{8 \epsilon_{0}^{3}}{\left(1-2 \epsilon_{0}\right)^{2}}
$$




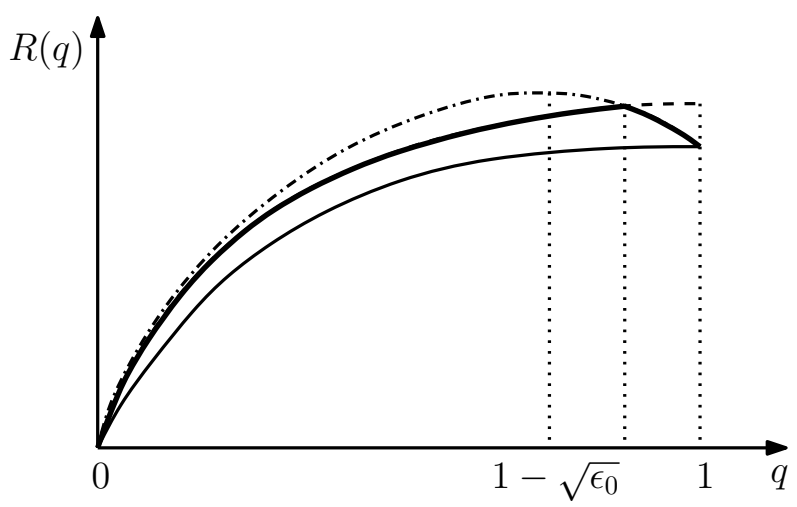

Figure 2: $R_{1}$ (the lower solid curve) is a quadratic curve that peaks at $q=1, R_{1}=1$, and passes through $q=0, R_{1}=0$. To construct $R_{2}$, first draw the revenue curves of the uniform distributions over $\left[1+\frac{2 \epsilon_{0}}{1-\sqrt{\epsilon_{0}}+2 \epsilon_{0}}, 2\right]$ (the dashed curve) and $\left[1,1+\frac{1}{1-2 \sqrt{\epsilon_{0}}}\right]$ (the dash-dotted curve). $R_{2}$ (the bold solid curve) is the lower envelope of the two curves.

Proof. By $(7)$, we have $\left(1-2 \epsilon_{0}\right) \leq \frac{f_{1}}{f_{2}} \leq\left(1-2 \epsilon_{0}\right)^{-1}$. Further, note that a $1-2 \epsilon$ fraction (w.r.t. quantile) of $D_{1}$ and $D_{2}$ are identical. The lemma follows from Lemma 4.4.

Let $R_{1}$ and $R_{2}$ be the revenue curves of $D_{1}$ and $D_{2}$. Let $R_{1}^{*}$ and $R_{2}^{*}$ be the corresponding optimal revenues. The following lemmas follow directly from the definition of $D_{1}$ and $D_{2}$.

Lemma 4.10. $R_{1}(v) \geq\left(1-\epsilon_{0}\right) R_{1}^{*}$ if and only if $v \geq \frac{1}{\epsilon_{0}}-1$.

Lemma 4.11. $R_{2}(v) \geq\left(1-\epsilon_{0}\right) R_{1}^{*}$ if and only if $\frac{1}{\epsilon_{0}}-3+2 \epsilon_{0} \geq v \geq \frac{1}{2 \epsilon_{0}-\epsilon_{0}^{2}}-1$.

Recall that $\epsilon_{0}=3 \epsilon$. The $(1-3 \epsilon)$-optimal price sets of $D_{1}$ and $D_{2}$ are disjoint. Theorem 4.8 follows from Theorem 4.2 and Lemma 4.9.

MHR Lower Bound We now turn to MHR distributions and show that $(1-\epsilon)$-approximate pricing for MHR distributions requires $\Omega\left(\epsilon^{-3 / 2}\right)$ samples.

Theorem 4.12. Any pricing algorithm that is $(1-\epsilon)$-approximate for all MHR distributions uses at least $\Omega\left(\epsilon^{-3 / 2}\right)$ samples.

We again describe the two distributions used and defer the full proof to the Appendix. Let $D_{1}$ be the uniform distribution over $[1,2]$. Let $\epsilon_{0}=c \epsilon$ where $c$ is a sufficiently large constant to be determined later. Define $D_{2}$ by scaling up the density (of $D_{1}$ ) in $v \in\left[1+\sqrt{\epsilon_{0}}, 2\right]$ by a factor of $1+\frac{2 \epsilon_{0}}{1-\sqrt{\epsilon_{0}}}$ and scaling down the density in $v \in\left[1,1+\sqrt{\epsilon_{0}}\right]$ by a factor $1-2 \sqrt{\epsilon_{0}}$, i.e.,

$$
f_{2}(v)= \begin{cases}1-2 \sqrt{\epsilon_{0}} & \text { if } 1 \leq v \leq 1+\sqrt{\epsilon_{0}} \\ 1+\frac{2 \epsilon_{0}}{1-\sqrt{\epsilon_{0}}} & \text { if } 1+\sqrt{\epsilon_{0}}<v \leq 2\end{cases}
$$

We summarize the revenue curves of $D_{1}$ and $D_{2}$ in Figure 2. 


\section{Single Sample Regime: Beating Identity Pricing for MHR Dis- tributions}

This section considers deterministic 1-sample pricing strategies. Recall from the Introduction that "identity pricing," meaning $p\left(v_{i}\right)=v_{1}$, has an approximation guarantee of $\frac{1}{2}$ for the class of regular distributions. We show in the appendix that there is no better 1-sample deterministic pricing strategy for the class of regular distributions, and that identity pricing is no better than $\frac{1}{2}$-approximate even for the special case of MHR distributions.

Our next goal is to show that scaling down the sampled value, i.e., $p(v)=c v$ for some constant $c<1$, achieves an approximation ratio better than $\frac{1}{2}$ for MHR distributions.

Theorem 5.1. $p(v)=0.85 v$ is 0.589-approximate for MHR distributions.

The intuition is as follows. We divide the quantile space into two subsets: those that are larger than the quantile of the optimal reserve, i.e., $q^{*}$, and those that are smaller.

- First, consider those that are larger. We recall the argument that identity pricing is $\frac{1}{2}$ approximate: the expected revenue of identity pricing is the area under the revenue curve; by concavity of the revenue curve, this is at least half the height and, thus, half the optimal revenue. We show that the revenue curve of an MHR distribution is at least as concave as that of an exponential distribution (Lemma C.2). This implies that identity pricing is strictly better than $\frac{1}{2}$-approximate for quantiles larger than $q^{*}$. Furthermore, scaling down the price by a factor of $c$ decreases the revenue by at most a factor of $c$. For $c<1$ close enough to 1 , the expected revenue of this part is still strictly better than one half of the optimal.

- Next, consider quantiles that are smaller than $q^{*}$. Theorem C.1 suggests that an approximate point mass is a worst-case scenario, where the sale probability of identity pricing is only $\frac{1}{2}$ on average. By scaling down the sampled value by a little, we double the selling probability w.r.t. a point mass without changing the price by much. So the expected revenue of this part is also strictly better than one half of optimal.

In the Appendix, we present the formal argument.

\section{Single Sample Negative Results}

First, we note that identify pricing is an optimal deterministic strategy for regular distributions (see Appendix for all proofs).

Theorem 6.1. No deterministic 1-pricing strategy is better than a $\frac{1}{2}$-approximation for regular distributions.

Next, we turn to MHR distributions. We first present a negative result that holds for every continuously differentiable pricing. Then, we present a slightly weaker negative result that holds for all deterministic 1-pricing algorithms.

Theorem 6.2. No continuously differentiable 1-pricing strategy is better than a 0.677-approximate for MHR distributions.

We consider exponential distributions and truncated exponential distributions, i.e., truncate the all values that are higher than some threshold $v^{*}$ in an exponential distribution and replace it with a uniform distribution over $\left[v^{*}, v^{*}+\alpha\right]$, where $v^{*}$ and $\alpha$ are parameters to be determined 
later. We will show that no continuously differentiable pricing functions can achieve better than 0.677-approximation in both cases. We consider these two distributions because of the intuition from the MHR upper bound analysis: the algorithm need to scale down the sampled value to handle point mass distributions, but it should not scale down the value by too much, so that it still gest good revenue for, e.g., the exponential distribution. The truncation is an approximation to a point mass and forces the algorithm to scale down.

Theorem 6.3. No deterministic 1-pricing strategy is better than a $\frac{e}{4} \approx 0.68$-approximate for $M H R$ distributions.

To prove this more general negative result, we consider a distribution of exponential distributions, i.e., we first draw a parameter $\lambda$ from a distribution and then draw the sample $v$ from an exponential distribution with parameter $\lambda$. We next solve for the best response pricing strategy w.r.t. this distribution of instances, which is the identity pricing. The theorem then follows from the approximation ratio of identity pricing for exponential distributions.

\section{References}

[1] M. Anthony and P. L. Bartlett. Neural Network Learning: Theoretical Foundations. Cambridge University Press, 1999.

[2] P. Azar, C. Daskalakis, S. Micali, and S. M. Weinberg. Optimal and efficient parametric auctions. In Proceedings of the 24th Annual ACM-SIAM Symposium on Discrete Algorithms (SODA), pages 596-604, 2013.

[3] Thomas Baigneres, Pascal Junod, and Serge Vaudenay. How far can we go beyond linear cryptanalysis? In Advances in Cryptology-Asiacrypt 2004, pages 432-450. 2004.

[4] Maria-Florina Balcan, Avrim Blum, Jason D. Hartline, and Yishay Mansour. Reducing mechanism design to algorithm design via machine learning. J. Comput. Syst. Sci., 74(8):1245-1270, 2008 .

[5] Sandeep Baliga and Rakesh Vohra. Market research and market design. Advances in Theoretical Economics, 3(1), 2003.

[6] Jeremy Bulow and Paul Klemperer. Auctions versus negotiations. The American Economic Review, 86(1):180-194, 1996.

[7] Shuchi Chawla, Jason D. Hartline, and Denis Nekipelov. Mechanism design for data science. In $E C$, pages $711-712,2014$.

[8] Alessandro Chiesa, Silvio Micali, and Zeyuan Allen Zhu. Mechanism design with approximate valuations. In Proceedings of the Innovations in Theoretical Computer Science Conference, pages $34-38,2012$.

[9] Richard Cole and Tim Roughgarden. The sample complexity of revenue maximization. In STOC, pages 243-252, 2014.

[10] Jacques Crémer and Richard P. McLean. Optimal selling strategies under uncertainty for a discriminating monopo list when demands are interdependent. Econometrica, 53(2):345-361, 1985 . 
[11] Peerapong Dhangwatnotai, Tim Roughgarden, and Qiqi Yan. Revenue maximization with a single sample. In EC, pages 129-138, 2010.

[12] Cynthia Dwork, Guy N. Rothblum, and Salil Vadhan. Boosting and differential privacy. In FOCS, pages 51-60. IEEE, 2010.

[13] Hu Fu, Nima Haghpanah, Jason D. Hartline, and Robert Kleinberg. Optimal auctions for correlated buyers with sampling. In EC, pages 23-36, 2014.

[14] Andrew V Goldberg, Jason D Hartline, Anna R Karlin, Michael Saks, and Andrew Wright. Competitive auctions. Games and Economic Behavior, 55(2):242-269, 2006.

[15] J. D. Hartline and T. Roughgarden. Optimal platform design. arXiv:1412.8518, 2014.

[16] Jason Hartline, Vahab Mirrokni, and Mukund Sundararajan. Optimal marketing strategies over social networks. In $W W W$, pages 189-198, 2008.

[17] Jason D. Hartline. Mechanism design and approximation. Book draft, 2014.

[18] Zvika Neeman. The effectiveness of English auctions. Games and Economic Behavior, 43(2): 214-238, 2003.

[19] Michael Ostrovsky and Michael Schwarz. Reserve prices in internet advertising auctions: A field experiment. In EC, pages 59-60, 2011.

[20] Mark S. Pinsker. Information and information stability of random variables and processes. Izv. Akad. Nauk, 1960.

[21] Ilya Segal. Optimal pricing mechanisms with unknown demand. The American Economic Review, 93(3):509-529, 2003.

[22] Balasubramanian Sivan and Vasilis Syrgkanis. Vickrey auctions for irregular distributions. In WINE, pages 422-435, 2013.

[23] Leslie G. Valiant. A theory of the learnable. Communications of the ACM, 27(11):1134-1142, 1984.

\section{A Missing Proofs from Section 3}

Proof of Theorem 3.3. We use the same setup and notation as in the MHR case. Using the weaker lemmas, we have $q_{i}, \tilde{q}_{i}=\Omega\left(\alpha^{1 /(1-\alpha)}\right)$, and $\Delta \geq \Omega\left(\alpha \delta^{2}\right)$.

If $\tilde{q}_{1} v_{1} \geq \tilde{q}_{2} v_{2}$, then

$$
\frac{\tilde{q}_{1}}{\tilde{q}_{2}} \geq \frac{v_{2}}{v_{1}}=\frac{R\left(q_{2}\right)}{R\left(q_{1}\right)} \frac{q_{1}}{q_{2}}=(1-\Delta)^{-1} \frac{q_{1}}{q_{2}}=\frac{q_{1}}{q_{2}}+\Omega\left(\alpha^{1 /(1-\alpha)} \Delta\right) .
$$

So

$$
\begin{aligned}
\tilde{q}_{2}-\tilde{q}_{1} & =\left(1-\frac{\tilde{q}_{1}}{\tilde{q}_{2}}\right) \tilde{q}_{2} \leq\left(1-\frac{\tilde{q}_{1}}{\tilde{q}_{2}}\right)\left(1+\epsilon^{3 / 4}\right) q_{2} \\
& \leq\left(1-\frac{q_{1}}{q_{2}}-\Omega\left(\alpha^{1 /(1-\alpha)} \Delta\right)\right)\left(1+\epsilon^{3 / 4}\right) q_{2}=q_{2}-q_{1}+\delta \epsilon^{3 / 4}-\Omega\left(\alpha^{1 /(1-\alpha)} \Delta\right) .
\end{aligned}
$$


Since $\delta=O(\sqrt{\Delta / \alpha})$ and $\Delta \geq \frac{\epsilon}{2}$, we have $\delta \epsilon^{3 / 4}=o(\Delta)$. So

$$
\tilde{q}_{2}-\tilde{q}_{1} \leq q_{2}-q_{1}-\Omega\left(\alpha^{1 /(1-\alpha)} \Delta\right) .
$$

That is, the number of samples that fall between $q_{1}$ and $q_{2}$ differs from its expectation by at least $\Omega\left(\alpha^{1 /(1-\alpha)} \Delta m\right)$. By Chernoff bound, the probability of this event is at most $\exp \left(-\frac{\left(\alpha^{1 /(1-\alpha)} \Delta\right)^{2} m}{\delta}\right)$. Recall that $\Delta=\Omega\left(\alpha \delta^{2}\right), \Delta \geq \frac{\epsilon}{2}$, and $m=\Theta\left(\alpha^{-2 /(1-\alpha)-1 / 2} \epsilon^{-3 / 2} \log \epsilon^{-1}\right)$. So this probability is at most $\exp \left(-\Omega\left(\log \epsilon^{-1}\right)\right)=o\left(\frac{1}{m^{2}}\right)$ with a suitable choice of parameters.

\section{B Missing Proofs from Section 4}

Proof of Lemma 4.3. By the definition of KL divergence, we have

$$
\begin{aligned}
D_{K L}\left(D_{1} \| D_{2}\right)+D_{K L}\left(D_{2} \| D_{1}\right) & =\int_{\Omega}\left[p_{1}(\omega) \ln \frac{p_{1}(\omega)}{p_{2}(\omega)}+p_{2}(\omega) \ln \frac{p_{2}(\omega)}{p_{1}(\omega)}\right] d \omega \\
& =\int_{\Omega}\left[p_{1}(\omega)\left(\ln \frac{p_{1}(\omega)}{p_{2}(\omega)}+\ln \frac{p_{2}(\omega)}{p_{1}(\omega)}\right)+\left(p_{2}(\omega)-p_{1}(\omega)\right) \ln \frac{p_{2}(\omega)}{p_{1}(\omega)}\right] d \omega \\
& \leq \int_{\Omega}\left[0+\left|p_{2}(\omega)-p_{1}(\omega)\right| \ln (1+\epsilon)\right] d \omega
\end{aligned}
$$

The last inequality follows by $(1+\epsilon)^{-1} \leq \frac{f_{1}(\omega)}{f_{2}(\omega)} \leq(1+\epsilon)$. Further, this condition also implies that

$$
\left|p_{2}(\omega)-p_{1}(\omega)\right| \leq((1+\epsilon)-1) \min \left\{p_{1}(\omega), p_{2}(\omega)\right\}=\epsilon \min \left\{p_{1}(\omega), p_{2}(\omega)\right\} .
$$

Thus, we have

$$
D_{K L}\left(D_{1} \| D_{2}\right)+D_{K L}\left(D_{2} \| D_{1}\right) \leq \epsilon \ln (1+\epsilon) \int_{\Omega} \min \left\{p_{1}(\omega), p_{2}(\omega)\right\} d \omega \leq \epsilon \ln (1+\epsilon) \leq \epsilon^{2} .
$$

\section{B.1 Proof of Theorem 4.12}

Recall that the revenue curves of $D_{1}$ and $D_{2}$ are summarized in Figure 2.

\section{Lemma B.1.}

$$
D_{K L}\left(D_{1} \| D_{2}\right)+D_{K L}\left(D_{2} \| D_{1}\right)=O\left(\epsilon_{0}^{3 / 2}\right) .
$$

Proof. By our choice of $D_{1}$ and $D_{2}, D_{1}$ and $D_{2}$ differs by $1+\frac{2 \epsilon_{0}}{1-\sqrt{\epsilon_{0}}}$ for $v \in\left[1+\sqrt{\epsilon_{0}}, 2\right]$, and differs by $1-2 \sqrt{\epsilon_{0}}$ for $v \in\left[1,1+\sqrt{\epsilon_{0}}\right]$. The lemma follows from Lemma 4.5.

Let $q_{i}^{*}$ be the revenue optimal quantile of $R_{i}$, and let $R_{i}^{*}=R_{i}\left(q_{i}^{*}\right)$ be the optimal revenue.

Lemma B.2. $q_{1}^{*}=1$, and $R_{1}^{*}=1$.

Proof. $R_{1}(q)=q(2-q)$ is a quadratic curve that peaks at $q=1$ with $R_{1}(1)=1$.

Lemma B.3. $q_{2}^{*}=1-\sqrt{\epsilon_{0}}+2 \epsilon_{0}$, and $R_{2}^{*}=1+\epsilon_{0}+2 \epsilon^{3 / 2}$. 
Proof. For $1 \leq v \leq 1+\sqrt{\epsilon_{0}}$ and $1-\sqrt{\epsilon_{0}}+2 \epsilon_{0} \leq q(v) \leq 1, R_{2}$ is identical to the revenue curve of the uniform distribution over $\left[1,1+\frac{1}{1-2 \sqrt{\epsilon_{0}}}\right]$, i.e., $q\left(\frac{2-2 \sqrt{\epsilon_{0}}}{1-2 \sqrt{\epsilon_{0}}}-\frac{1}{1-2 \sqrt{\epsilon_{0}}} q\right)$, which is maximized at $q=1-\sqrt{\epsilon_{0}}$.

For $1+\sqrt{\epsilon_{0}} \leq v \leq 2$ and $0 \leq q(v) \leq 1-\sqrt{\epsilon_{0}}+2 \epsilon_{0}, R_{2}$ is identical to the revenue curve of the uniform distribution over $\left[1+\frac{2 \epsilon_{0}}{1-\sqrt{\epsilon_{0}}+2 \epsilon_{0}}, 2\right]$, i.e., $q\left(2-\frac{1-\sqrt{\epsilon_{0}}}{1-\sqrt{\epsilon_{0}}+2 \epsilon_{0}} q\right)$, which is maximized at $q=1$ in interval $[0,1]$.

$R_{2}$ is maximized at the intersection of the two parts, where $q=1-\sqrt{\epsilon_{0}}+2 \epsilon_{0}$, and $R_{2}\left(1-\sqrt{\epsilon_{0}}+\right.$ $\left.2 \epsilon_{0}\right)=1+\epsilon_{0}+2 \epsilon^{3 / 2}$.

The following lemmas follow from our construction of $D_{1}$ and $D_{2}$.

Lemma B.4. If $R_{1}(q) \geq(1-3 \epsilon) R_{1}^{*}$, then $q \geq 1-\sqrt{3 \epsilon}$ and $v(q) \leq \frac{1-3 \epsilon}{1-\sqrt{3 \epsilon}} R_{1}^{*}$.

Lemma B.5. If $R_{2}(q) \geq(1-3 \epsilon) R_{2}^{*}$, then $q \leq 1-\sqrt{\epsilon_{0}}+2 \epsilon_{0}+\sqrt{3 \epsilon}$ and $v(q) \geq \frac{1-3 \epsilon}{1-\sqrt{\epsilon_{0}}+2 \epsilon_{0}+\sqrt{3 \epsilon}} R_{2}^{*}$.

Therefore, when $\epsilon_{0}=c \epsilon$ for sufficiently large constant $c$, we have $1-\sqrt{3 \epsilon}>1-\sqrt{\epsilon_{0}}+2 \epsilon_{0}+\sqrt{3 \epsilon}$. Further note that $R_{2}^{*}>R_{1}^{*}$, so the $(1-3 \epsilon)$-optimal price sets of $D_{1}$ and $D_{2}$ are disjoint. Theorem 4.12 then follows from Theorem 4.2 and Lemma B.1.

\section{Missing Proofs from Section 5}

\section{C.1 Lower Bound for Identity Pricing}

Since MHR distributions are more restrictive than regular ones, one might expect identity pricing to give better than $\frac{1}{2}$-approximation for then. This is not the case.

Theorem C.1. The identity pricing algorithm is no better than $\frac{1}{2}$-approximation for MHR distributions.

Proof. Consider the uniform distribution over $[1-\epsilon, 1]$. The optimal revenue is $1-\epsilon$, with reserve price $1-\epsilon$. The identity pricing algorithm gets revenue $\frac{1}{\epsilon^{2}} \int_{1-\epsilon}^{1} v(1-v) d v=\frac{1}{2}-\frac{1}{3} \epsilon$. The approximation ratio approaches $\frac{1}{2}$ as $\epsilon$ goes to zero.

We remark that the lower bound still holds if the support must start from 0 because we can add a little mass on $[0,1-\epsilon]$ without changing the nature of the lower bound. The same trick applies to the lower bounds in Section 6.

\section{C.2 Proof of Theorem 5.1}

Let $\hat{R}(q)$ be the expected revenue of reserve price $p(v(q)))=c v(q)$ w.r.t. revenue curve $R$. Define $\hat{R}^{\exp }(q)$ similarly w.r.t. the exponential distribution $D^{\exp }$. For ease of presentation, we will w.l.o.g. scale the values so that $v\left(q^{*}\right)=v^{\exp }\left(q^{*}\right)$ and, thus, $R\left(q^{*}\right)=R^{\exp }\left(q^{*}\right)$.

\section{Technical Lemmas about MHR Distributions}

Given the revenue at quantile $q^{*}, R^{\exp }$ minimizes the revenue at any quantile larger than $q^{*}$ among all MHR distributions.

Lemma C.2. For any $1 \geq q \geq q^{*}$, then $R(q) \geq R^{\exp }(q)$. 
Proof. Suppose not. Let $q_{0}$ be a quantile such that $R\left(q_{0}\right)<R^{\exp }\left(q_{0}\right)$. Since $R\left(q^{*}\right)=R^{\exp }\left(q^{*}\right)$ and that $R$ and $R^{\exp }$ are continuous, there exists $q_{1} \in\left[q^{*}, q_{0}\right]$ such that $R\left(q_{1}\right)=R^{\exp }\left(q_{1}\right)$ and $R^{\prime}\left(q_{1}\right) \leq\left(R^{\exp }\right)^{\prime}\left(q_{1}\right)$. Similarly, since $R(1) \geq 0=R^{\exp }(1)$, there exists $q_{2} \in\left[q_{0}, 1\right]$ such that $R\left(q_{2}\right)<R^{\exp }\left(q_{2}\right)$ and $R^{\prime}\left(q_{2}\right)>\left(R^{\exp }\right)^{\prime}\left(q_{2}\right)$.

Recall that $R^{\prime}\left(q_{1}\right)=\phi\left(v\left(q_{1}\right)\right)$ and $R^{\prime}\left(q_{2}\right)=\phi\left(v\left(q_{2}\right)\right)$. By the MHR assumption,

$$
R^{\prime}\left(q_{1}\right)-R^{\prime}\left(q_{2}\right)=\phi\left(v\left(q_{1}\right)\right)-\phi\left(v\left(q_{2}\right)\right) \geq v\left(q_{1}\right)-v\left(q_{2}\right)=\frac{R\left(q_{1}\right)}{q_{1}}-\frac{R\left(q_{2}\right)}{q_{2}} .
$$

By the definition of $R^{\exp }$, the above relation holds with equality for $R^{\exp }$ :

$$
\left(R^{\exp }\right)^{\prime}\left(q_{1}\right)-\left(R^{\exp }\right)^{\prime}\left(q_{2}\right)=\frac{R^{\exp }\left(q_{1}\right)}{q_{1}}-\frac{R^{\exp }\left(q_{2}\right)}{q_{2}}
$$

By (9) minus (10) and that $R\left(q_{1}\right)=R^{\exp }\left(q_{1}\right)$, we have

$$
R^{\prime}\left(q_{1}\right)-R^{\prime}\left(q_{2}\right)-\left(R^{\exp }\right)^{\prime}\left(q_{1}\right)+\left(R^{\exp }\right)^{\prime}\left(q_{2}\right) \geq \frac{R^{\exp }\left(q_{2}\right)}{q_{2}}-\frac{R\left(q_{2}\right)}{q_{2}}>0
$$

contradicting $R^{\prime}\left(q_{1}\right) \leq\left(R^{\exp }\right)^{\prime}\left(q_{1}\right)$ and $R^{\prime}\left(q_{2}\right)>\left(R^{\exp }\right)^{\prime}\left(q_{2}\right)$.

The above lemma does not apply to quantiles smaller than $q^{*}$. Consider a point mass, or a uniform distribution over $[1-\epsilon, 1]$ for sufficiently small $\epsilon$ for a counter example. The problem with applying it to quantiles smaller than $q^{*}$ is that $q_{1}$ might be 0 . So we have zero divided by zero issue in (9) and (10) and, thus, $R\left(q_{1}\right) / q_{1}$ may not equal $R^{\exp }\left(q_{1}\right) / q_{1}$ when we subtract (10) from (9).

As a corollary of Lemma C.2, $R^{\text {exp }}$ minimizes the value at each quantile larger than $q^{*}$ (Lemma C.3), and it maximizes the quantile at each value smaller than $v\left(q^{*}\right)$ (Lemma C.4).

Lemma C.3. For any $1 \geq q \geq q^{*}$, then $v(q) \geq v^{\exp }(q)$.

Lemma C.4. For any $0 \leq v \leq v\left(q^{*}\right)$, then $q(v) \geq q^{\exp }(v)$.

\section{Expected Revenue of Large Quantiles}

We show that for quantiles between $q^{*}$ and $1, R^{\exp }$ is indeed the worst-case scenario.

Lemma C.5. For any $1 \geq q \geq q^{*}, \hat{R}(q) \geq \hat{R}^{\exp }(q)$.

Proof. We abuse notation and let $R(v)=R(q(v))$. By Lemma C.3, $v(q) \geq v^{\exp }(q)$. So we have $c v(q) \geq c v^{\exp }(q)$. Since $R(v)$ is non-decreasing when $v>v\left(q^{*}\right)$ and $v\left(q^{*}\right)>c v(q) \geq c v^{\exp }(q)$, we have $\hat{R}(q)=R(c v(q)) \geq R\left(c v^{\exp }(q)\right)$.

Further, by Lemma C.4, $R^{\exp }$ minimizes the sale probability at any price $v \leq v\left(q^{*}\right)$ and, thus, minimizes the revenue at price $v$. So we have $R\left(c v^{\exp }(q)\right) \geq R^{\exp }\left(c v^{\exp }(q)\right)=\hat{R}^{\exp }(q)$ and the lemma follows.

As direct corollary of Lemma C.5, we can lower bound the expected revenue of quantiles between $q^{*}$ and 1 by the expected revenue of the worst-case distribution $R^{\exp }$.

Lemma C.6. $\int_{q^{*}}^{1} \hat{R}(q) d q \geq \int_{q^{*}}^{1} \hat{R}^{\exp }(q) d q$. 


\section{Expected Revenue of Small Quantiles}

Next, we consider quantiles between 0 and $q^{*}$. Let $q_{0}$ be that $c v\left(q_{0}\right)=v\left(q^{*}\right)$, i.e., the reserve price is smaller than $v\left(q^{*}\right)$ if and only if the sample has quantile larger than $q_{0}$. We will first lower bound the expected revenue of quantiles smaller than $q_{0}$, and then handle the other quantiles.

Lemma C.7. For any $0 \leq q^{\prime} \leq q_{0}, \int_{0}^{q^{\prime}} \hat{R}(q) d q \geq \frac{1+\sqrt{1-c}}{2} q^{\prime} R\left(q^{\prime}\right)$.

Proof. Let $c_{0}=\frac{c}{2}$. For any $i \geq 0$, let $c_{i+1}=1-\frac{c}{4 c_{i}}$. We will inductively show that $\int_{0}^{q^{\prime}} \hat{R}(q) d q \geq$ $c_{i} q^{\prime} R\left(q^{\prime}\right)$, and then prove that $c_{i}$ converges to $\frac{1+\sqrt{1-c}}{2}$.

Base Case By concavity of the revenue curve, $\int_{0}^{q^{\prime}} R(q) d q \geq \frac{1}{2} R\left(q^{\prime}\right)$. Further, lower reserve prices has larger sale probability. So $\hat{R}(q) \geq c R(q)$ and the base case follows.

Inductive Step Let $q_{1}$ be that $c v\left(q_{1}\right)=v\left(q^{\prime}\right)$, i.e., the reserve price is smaller than $v\left(q^{\prime}\right)$ iff the sample has quantile larger than $q_{1}$. We have

$$
R\left(q_{1}\right)=v\left(q_{1}\right) q_{1}=\frac{v\left(q^{\prime}\right) q_{1}}{c}=\frac{q_{1}}{c q^{\prime}} q^{\prime} v\left(q^{\prime}\right)=\frac{q_{1}}{c q^{\prime}} R\left(q^{\prime}\right)
$$

For the interval from 0 to $q_{1}$, by inductive hypothesis we have

$$
\int_{0}^{q_{1}} \hat{R}(q) d q \geq c_{i} q_{1} R\left(q_{1}\right)=\frac{c_{i} q_{1}^{2}}{c q^{\prime}} R\left(q^{\prime}\right)
$$

Next, consider the interval from $q_{1}$ to $q^{\prime}$. For any $q_{1} \leq q \leq q^{\prime}$, by that $q^{\prime} \leq q_{0}$ and our choice of $q_{0}$, we have $v\left(q^{*}\right)<c v(q)<c v\left(q_{1}\right)=v\left(q^{\prime}\right)$. So $\hat{R}(q) \geq R\left(q^{\prime}\right)$. Thus,

$$
\int_{q_{1}}^{q^{\prime}} \hat{R}(q) d q \geq\left(q^{\prime}-q_{1}\right) R\left(q^{\prime}\right) .
$$

Putting together, we have

$$
\int_{0}^{q^{\prime}} \hat{R}(q) d q \geq\left(\frac{c_{i}}{c}\left(\frac{q_{1}}{q^{\prime}}\right)^{2}+\left(1-\frac{q_{1}}{q^{\prime}}\right)\right) q^{\prime} R\left(q^{\prime}\right) .
$$

Minimizing the RHS over $0 \leq q_{1} \leq q^{\prime}$, we have

$$
\int_{0}^{q^{\prime}} \hat{R}(q) d q \geq\left(1-\frac{c}{4 c_{i}}\right) q^{\prime} R\left(q^{\prime}\right)=c_{i+1} q^{\prime} R\left(q^{\prime}\right) .
$$

Convergence of $c_{i}$ There is only one stable stationary point, $\frac{1+\sqrt{1-c}}{2}$, for the recursion $c_{i+1}=$ $1-\frac{c}{4 c_{i}}$. The other, non-stable, stationary point is $\frac{1-\sqrt{1-c}}{2}$. Note that for any $0<c<1, c_{0}=\frac{c}{2}>$ $\frac{1-\sqrt{1-c}}{2}$. So $c_{i}$ converges to $\frac{1+\sqrt{1-c}}{2}$ as $i$ goes to infinity.

Lemma C.8. If $c=0.85$, then $\int_{0}^{q^{*}} \hat{R}(q) d q \geq 0.656 q^{*} R\left(q^{*}\right)$. 
Proof. Recall that $c v\left(q_{0}\right)=v\left(q^{*}\right)$. So

$$
R\left(q_{0}\right)=q_{0} v\left(q_{0}\right)=\frac{q_{0}}{c q^{*}} q^{*} v\left(q^{*}\right)=\frac{q_{0}}{c q^{*}} R\left(q^{*}\right) .
$$

Plugging $c=0.85$ and $\frac{1+\sqrt{1-c}}{2} \geq 0.693$ into Lemma C.7, we have

$$
\int_{0}^{q_{0}} \hat{R}(q) d q \geq 0.693 q_{0} R\left(q_{0}\right)=0.693 \frac{1}{c}\left(\frac{q_{0}}{q^{*}}\right)^{2} q^{*} R\left(q^{*}\right) \geq 0.815\left(\frac{q_{0}}{q^{*}}\right)^{2} q^{*} R\left(q^{*}\right) .
$$

On the other hand, for every $q_{0} \leq q \leq q^{*}$, by concavity of the revenue curve, we have

$$
q v(q) \geq \frac{q-q_{0}}{q^{*}-q_{0}} q^{*} v\left(q^{*}\right)+\frac{q^{*}-q}{q^{*}-q_{0}} q_{0} v\left(q_{0}\right) .
$$

Thus,

$$
v(q) \geq \frac{q^{*} v\left(q^{*}\right)-q_{0} v\left(q_{0}\right)}{q^{*}-q_{0}}+\frac{1}{q} \frac{q_{0} q^{*}}{q^{*}-q_{0}}\left(v\left(q_{0}\right)-v\left(q^{*}\right)\right) .
$$

Further, by our choice of $q_{0}$, the quantile of $c v(q)$ is at least $q^{*}$. So we have

$$
\hat{R}(q) \geq c v(q) q^{*} \geq\left(\frac{q^{*} v\left(q^{*}\right)-q_{0} v\left(q_{0}\right)}{q^{*}-q_{0}}+\frac{1}{q} \frac{q_{0} q^{*}}{q^{*}-q_{0}}\left(v\left(q_{0}\right)-v\left(q^{*}\right)\right)\right) c q^{*} .
$$

Let $x=\frac{q_{0}}{q^{*}}$. Plugging in $c v\left(q_{0}\right)=v\left(q^{*}\right), R\left(q_{0}\right)=\frac{q_{0}}{c q^{*}} R\left(q^{*}\right)$, and $c=0.85$, we have

$$
\left(\frac{0.85-x}{q^{*}-q_{0}}+\frac{1}{q} \frac{(1-0.85) x}{1-x}\right) q^{*} R\left(q^{*}\right) .
$$

Integrating over $q$ from $q_{0}$ to $q^{*}$, we have

$$
\int_{q_{0}}^{q^{*}} \hat{R}(q) d q \geq\left((0.85-x)+\ln \left(\frac{1}{x}\right) \frac{0.15 x}{1-x}\right) q^{*} R\left(q^{*}\right) .
$$

Summing up (11) and (12) gives

$$
\int_{0}^{q^{*}} \hat{R}(q) d q \geq\left((0.85-x)+\ln \left(\frac{1}{x}\right) \frac{0.15 x}{1-x}+0.815 x^{2}\right) q^{*} R\left(q^{*}\right) .
$$

We would like to minimize $f(x)$ for $x \in[0,1]$, where

$$
f(x)=(0.85-x)+\ln \left(\frac{1}{x}\right) \frac{0.15 x}{1-x}+0.815 x^{2} .
$$

Taking the derivative we have

$$
f^{\prime}(x)=-1-\frac{0.15}{1-x}-\frac{0.15 \ln (x)}{(1-x)^{2}}+1.63 x .
$$

This function has two roots in $x \in[0,1]$, at $x \in[0.546,0.547]\left(f^{\prime}(0.546)<-3 * 10^{-5}\right.$ and $f^{\prime}(0.547)>$ $\left.10^{-3}\right)$ and at $x \in\left[4.7 \cdot 10^{-4}, 4.8 \cdot 10^{-4}\right]\left(f^{\prime}(4.7 \cdot 10-4)>1.3 \cdot 10^{-3}\right.$ and $\left.f^{\prime}\left(4.8 \cdot 10^{-4}\right)<1.9 \cdot 10^{-3}\right)$. Testing the second derivative

$$
f^{\prime \prime}(x)=-\frac{0.15}{(1-x)^{2}}-\frac{0.3 \ln (x)}{(1-x)^{3}}-\frac{0.15}{x(1-x)^{2}}+1.63
$$

we have that $x \approx 0.546$ is a minimum point $\left(f^{\prime \prime}(0.546) \approx 1.5\right)$ and $x \approx 4.7 * 10^{-4}$ is a local maximum $\left(f^{\prime \prime}\left(4.7 * 10^{-4}\right) \approx-315\right)$. Therefore, the only remaining point we need to test is $x=0$ (the end of the interval $[0,1])$ and we have $\lim _{x \rightarrow 0} f(x)=0.85$. 


\section{Proof of Theorem 5.1}

Plugging in the exponential distribution in Lemma C.6, we obtain

$$
\int_{q^{*}}^{1} \hat{R}(q) d q \geq \frac{c}{(c+1)^{2}} \frac{1-\left(q^{*}\right)^{c+1}+\left(q^{*}\right)^{c+1} \ln \left(q^{*}\right)^{c+1}}{-\left(q^{*}\right) \ln \left(q^{*}\right)} R\left(q^{*}\right) .
$$

Combining this with Lemma C.8 yields

$$
\int_{0}^{1} \hat{R}(q) d q \geq\left(0.656 q^{*}+\frac{c}{(c+1)^{2}} \frac{1-\left(q^{*}\right)^{c+1}+\left(q^{*}\right)^{c+1} \ln \left(q^{*}\right)^{c+1}}{-\left(q^{*}\right) \ln \left(q^{*}\right)}\right) R\left(q^{*}\right) .
$$

We would like to lower bound $f(x)$, where

$$
f(x)=0.656 x+\frac{c}{(c+1)^{2}}\left(-x^{-1} \ln ^{-1}(x)+x^{c} \ln ^{-1}(x)-(c+1) x^{c}\right) .
$$

The derivative is

$f^{\prime}(x)=0.656+\frac{c}{(c+1)^{2}}\left(x^{-2} \ln ^{-1}(x)+x^{-2} \ln ^{-2}(x)+c x^{c-1} \ln ^{-1}(x)-x^{c-1} \ln ^{-2}(x)-c(c+1) x^{c}\right)$.

For $c=0.85$, we have a root at some $x \in[0.544,0.545]$ as $f^{\prime}(0.544)<-1.7 \cdot 10^{-4}$ and $f^{\prime}(0.545)>$ $9 \cdot 10^{-4}$. By testing the second derivative, this is a local minimum and the only root in the interval. The minimal value is at least 0.589 and the lemma follows.

\section{Missing Proofs in 6}

Proof of Theorem 6.1. Distributions with triangle revenue curves (with vertices $(0,0),(1,0)$, and $\left.\left(q^{*}, R\left(q^{*}\right)\right)\right)$ are commonly considered to be the worst-case regular distributions because they have the least concave revenue curves. In particular, we consider two such distributions: a point mass at $v$, whose revenue curve is a triangle with $\left(q^{*}, R\left(q^{*}\right)\right)=(1, v)$, and the distribution $F(v)=1-\frac{1}{v+1}$, whose revenue curve is a triangle with $\left(q^{*}, R\left(q^{*}\right)\right)=(0,1)$.

To achieve a non-trivial approximation ratio when the distribution is a point mass at $v, p(v) \leq v$ must hold. Then, consider the second distribution. The revenue at price $v$ is $\frac{v}{v+1}$, which is strictly increasing in $v$. So every deterministic pricing algorithm that satisfies $p(v) \leq v$ gets revenue less than or equal to that of the identity pricing algorithm $p(v)=v$, which is 2-approximate for the second distribution.

Proof of Theorem 6.2. Let us first assume the pricing algorithm is linear, i.e., $b(v)=c v$. As in the proof of Theorem 6.1, the algorithm has a finite approximation ratio only if $c \leq 1$.

We consider exponential distributions and truncated exponential distributions, i.e., truncate the all values that are higher than some threshold $v^{*}$ in an exponential distribution and replace it with a uniform distribution over $\left[v^{*}, v^{*}+\alpha\right]$, where $v^{*}$ and $\alpha$ are parameters to be determined later. We show that no scaling parameter $c$ can achieve better than 0.67-approximation in both cases.

We consider these two distributions because of the intuition from the MHR upper bound analysis: the algorithm needs to scale down the sampled value to handle point mass case, but it should not scale down the value by too much in order to get good revenue for, e.g., the exponential distribution. The truncation is an approximation to a point mass and forces the algorithm to scale down. 
Exponential Distribution Consider the approximation ratio w.r.t. the exponential distribution $D^{\exp }$. Given a sample with quantile $q$, its value is $v^{\exp }(q)=-\ln q$. So $b(v(q))=-c \ln q=-\ln q^{c}$ and the sale probability is $q^{c}$. The expected revenue of $b(v)$ is

$$
\int_{0}^{1}-q^{c} \ln q^{c} d q=\frac{c}{(c+1)^{2}}
$$

Recall that $R^{\exp }(q)=-q \ln q$, which is maximized at $q=\frac{1}{e}$ with optimal revenue $\frac{1}{e}$. So the approximation ratio w.r.t. $D^{\exp }$ is $\frac{e c}{(c+1)^{2}}$. Note that this immediately gives a lower bound of $\frac{e}{4} \approx 0.68$. If $c \leq 0.878$, then $\frac{e c}{(c+1)^{2}}<0.677$. For now on, we assume that $c \geq 0.878$.

Truncated Exponential Distribution Let $q^{*}=0.43$ and consider an exponential distribution such that $v\left(q^{*}\right)=1$. Truncate the exponential distribution at $q^{*}$ with a uniform distribution over $[1,1+\alpha]$ with $\alpha=0.74$. Hence, for $q \in\left[q^{*}, 1\right], v(q)=\frac{\ln q}{\ln q^{*}}$; for $q \in\left[0, q^{*}\right], v(q)=1+\alpha\left(1-\frac{q}{q^{*}}\right)$. It is easy to check that the revenue is maximized at $q=q^{*}$ with maximal revenue $q^{*}$.

Next, we upper bound the expected revenue of $b(v)$. Consider first the contribution from quantiles $q \in\left[\frac{1}{e}, 1\right]$. The analysis is similar to that of $D^{\exp }$, except that the values are scaled up by $-\frac{1}{\ln q^{*}}$. So this part contributes

$$
\begin{aligned}
-\frac{1}{\ln q^{*}} \int_{q^{*}}^{1}-q^{c} \ln q^{c} d q & =-\frac{1}{\ln q^{*}} \frac{c}{(c+1)^{2}}\left(1-\left(q^{*}\right)^{c+1}+\left(q^{*}\right)^{c+1} \ln \left(q^{*}\right)^{c+1}\right) \\
& \leq 2.7556 \frac{c}{(c+1)^{2}}\left(1-\left(q^{*}\right)^{c+1}+\left(q^{*}\right)^{c+1} \ln \left(q^{*}\right)^{c+1}\right) q^{*}
\end{aligned}
$$

Now consider the quantiles that are smaller than $q^{*}$ and have values at most $\frac{1}{c}$, i.e., the corresponding reserve price is smaller than 1 . These are quantiles $q \in\left[\frac{c \alpha+c-1}{c \alpha} q^{*}, q^{*}\right]$. We upper bound the expected revenue by the optimal revenue $q^{*}$ when the sampled quantile is in this interval. So this part contributes at most

$$
\left(q^{*}-\frac{c \alpha+c-1}{c \alpha} q^{*}\right) q^{*}=\left(\frac{1-c}{c \alpha}\right)\left(q^{*}\right)^{2} \leq 0.5811\left(\frac{1}{c}-1\right) q^{*} .
$$

Finally, consider quantiles $q \in\left[0, \frac{c \alpha+c-1}{c \alpha} q^{*}\right]$. The corresponding value is $v(q)=1+\alpha\left(1-\frac{q}{q^{*}}\right)$ and the reserve price is $b(v)=c\left(1+\alpha\left(1-\frac{q}{q^{*}}\right)\right)$. The sale probability at this price is $\frac{1+\alpha-b(v)}{\alpha} q^{*}$. So this part contributes

$$
\int_{0}^{\frac{c \alpha+c-1}{c \alpha} q^{*}} b(v) \frac{1+\alpha-b(v)}{\alpha} q^{*} d q .
$$

Note that $d b(v)=-\frac{c \alpha}{q^{*}} d q, b=1$ when $q=\frac{c \alpha+c-1}{c \alpha} q^{*}$, and $b=c(1+\alpha)$ when $q=0$. So the above equals

$$
\begin{aligned}
\frac{\left(q^{*}\right)^{2}}{c \alpha^{2}} \int_{0}^{c(1+\alpha)} b(v)(1+\alpha-b(v)) d b(v) & =\frac{\left(q^{*}\right)^{2}}{c \alpha^{2}}\left(\frac{1+\alpha}{2}\left((c(1+\alpha))^{2}-1\right)-\frac{1}{3}\left((c(1+\alpha))^{3}-1\right)\right) \\
& =\frac{\left(q^{*}\right)^{2}}{c \alpha^{2}}\left(\left(\frac{c^{2}}{2}-\frac{c^{3}}{3}\right)(1+\alpha)^{3}-\frac{1+\alpha}{2}+\frac{1}{3}\right) \\
& \leq\left(-1.3789 c^{2}+2.0683 c-0.4215 \frac{1}{c}\right) q^{*}
\end{aligned}
$$


Putting everything together and dividing the expected revenue by the optimal revenue $q^{*}$, the approximation ratio is at most

$2.7556 \frac{c}{(c+1)^{2}}\left(1-\left(q^{*}\right)^{c+1}+\left(q^{*}\right)^{c+1} \ln \left(q^{*}\right)^{c+1}\right)+0.5811\left(\frac{1}{c}-1\right)+\left(-1.3768 c^{2}+2.2683 c-0.4215 \frac{1}{c}\right)$.

We numerically maximize the above function over $c \in[0.878,1]$. It is decreasing in the interval $[0.878,1]$ and takes value about 0.6762 at $c=0.878$. So the lemma follows for linear pricing functions.

Continuously Differentiable Pricing Functions Next, we explain how to reduce the case of a continuously differentiable pricing function to the linear pricing function case. Since $b(v)$ is continuously differentiable, for any $\delta>0$, there exists $\epsilon>0$ such that for any $v \in[0, \epsilon]$, $\left|b^{\prime}(v)-b^{\prime}(0)\right|<\delta$, i.e., in this neighborhood of $0, b(v)$ behaves like a linear function with slope approximately $b^{\prime}(0)$ (up to error $\delta$ ). So we can handle them like the linear case.

Formally, if $b^{\prime}(0)<\frac{1}{2}$, then $b(v) \leq\left(\frac{1}{2}+\delta\right) v$ for $v \leq \epsilon$. So its approximation ratio w.r.t. a point mass at $v$ is at most $\frac{1}{2}+\delta<0.677$ for sufficiently small $\delta$.

Next, assume $b^{\prime}(0)>\frac{1}{2}$. Let us scale down the values in the exponential distribution and the truncated exponential distribution from the linear case such that all values are less than $\epsilon{ }^{12}$ For any sampled value $v$, the expected revenue is $b(v) q(b(v))$ where $\left(f^{\prime}(0)-\delta\right) v<b(v)<\left(f^{\prime}(0)+\delta\right) v$. So

$$
b(v) q(b(v)) \leq\left(f^{\prime}(0)+\delta\right) v q\left(\left(f^{\prime}(0)-\delta\right) v\right)=\frac{f^{\prime}(0)+\delta}{f^{\prime}(0)-\delta}\left(f^{\prime}(0)-\delta\right) v q\left(\left(f^{\prime}(0)-\delta\right) v\right),
$$

which is at most $\frac{f^{\prime}(0)+\delta}{f^{\prime}(0)-\delta}$ times larger than the revenue of linear pricing function $\left(f^{\prime}(0)-\delta\right) v$. The lemma then follows by $f^{\prime}(0) \geq \frac{1}{2}$ and letting $\delta$ goes to zero.

Proof of Theorem 6.3. By previous arguments, it suffices to consider only (deterministic) pricing functions with $p(v) \leq v$ for all $v$. We consider a distribution over value distributions. Draw $\lambda$ from an exponential distribution with parameter $\gamma$, i.e., the density of $\lambda$ is $\gamma e^{-\gamma \lambda}$, where $\gamma$ is a parameter to be determined later. Let the value distribution to be an exponential distribution with parameter $\lambda$, i.e., the density of $v$ is $\lambda e^{-\lambda v}$.

We first compute the best response pricing algorithm $p(v)$ subject to $p(v) \leq v$ for this case. The expected revenue of $p(v)$ is

$$
\begin{aligned}
R & =\int_{0}^{\infty}\left[\int_{0}^{\infty} \lambda e^{-\lambda v} p(v) e^{-\lambda p(v)} d v\right] \gamma e^{-\gamma \lambda} d \lambda \\
& =\gamma \int_{0}^{\infty}\left[\int_{0}^{\infty} \lambda e^{-\lambda(v+p(v)+\gamma)} p(v) d \lambda\right] d v \\
& =\gamma \int_{0}^{\infty}\left[\int_{0}^{\infty} \lambda e^{-\lambda(v+p(v)+\gamma)} p(v) d \lambda\right] d v \\
& =\gamma \int_{0}^{\infty}\left[\frac{p(v)}{v+p(v)+\gamma} \int_{0}^{\infty}(v+p(v)+\gamma) \lambda e^{-\lambda(v+p(v)+\gamma)} d \lambda\right] d v \\
& =\gamma \int_{0}^{\infty} \frac{p(v)}{(v+p(v)+\gamma)^{2}} d v .
\end{aligned}
$$

\footnotetext{
${ }^{12}$ Note that the support of the exponential distribution spans all non-negative real numbers. So instead of scaling to make all values smaller than $\epsilon$, we will make sure $1-10^{-5}$ fraction of the values are smaller than $\epsilon$; the remaining values can change the approximation ratio by at most $10^{-5}$.
} 
Note that $\frac{p(v)}{(v+p(v)+\gamma)^{2}}$ is maximized at $p(v)=v$ for $p(v) \leq v$. So the best response is $p(v)=v$. Given any $\lambda$, the optimal revenue is $\frac{1}{e \lambda}$, and the expected revenue of $p(v)=v$ is $\frac{1}{4 \lambda}$. So the approximation ratio is at most $\frac{e}{4} \approx 0.68$, as desired. 